\title{
Multicast-Based Loss Inference with Missing Data*
}

\author{
Nick Duffield ${ }^{\ddagger}$ Joseph Horowitz ${ }^{\star}$ Don Towsley ${ }^{\S}$ Wei Wei ${ }^{\S}$ Timur Friedman ${ }^{\S}$ \\ $\ddagger$ AT\&T Labs-Research \\ ๑Dept. Math. \& Statistics \\ ${ }^{\S}$ Dept. of Computer Science \\ 180 Park Avenue \\ Florham Park, NJ 07932, USA \\ University of Massachusetts \\ Amherst, MA 01003, USA \\ University of Massachusetts \\ Amherst, MA 01003, USA \\ duffield@ research.att.com \\ joeh@math.umass.edu \\ \{towsley,weiwei,friedman\}@cs.umass.edu
}

\begin{abstract}
Network tomography using multicast probes enables inference of loss characteristics of internal network links from reports of end-to-end loss seen at multicast receivers. In this paper we develop estimators for internal loss rates when reports are not available on some probes or from some receivers. This problem is motivated by the use of unreliable transport control protocols, such as RTCP, to transmit loss reports to a collector for inference. We use a maximum likelihood (ML) approach in which we apply the Expectation Maximization (EM) algorithm to provide an approximate value for the ML estimator for the incomplete data problem. We present a concrete implementation of the algorithm that can be applied to measured data. For certain classes of models we establish identifiability of the probe and report loss parameters, and convergence of the EM sequence to the MLE. Numerical results suggest that these properties hold more generally. We derive convergence rates for the EM iterates, and the estimation error of the MLE. Last, we evaluate the accuracy and convergence rate through extensive simulations.
\end{abstract}

Keywords: End-to-end Measurement, Network Tomography, Missing Data, Maximum Likelihood Estimation, EM Algorithm, Multicast, RTP, RTCP.

\section{Introduction}

\subsection{Motivation}

As the Internet grows in size and diversity, its internal performance becomes ever more difficult to measure. Any one organization has administrative access to only a small fraction of the network's internal nodes, whereas commercial factors often prevent organizations from sharing internal performance data.

One promising technique that avoids these problems, Multicast Inference of Network Characteristics (MINC), uses end-to-end multicast measurements to infer link-level loss rates and delay statistics by exploiting the inherent (and well characterized) correlation in performance observed by multicast receivers. These measurements do not rely on administrative access to internal nodes since the inference can be calculated using only information recorded at the end hosts.

The key intuition for inferring packet loss is that the arrival of a packet at a given internal node can be directly inferred from the packet's arrival at one or more receivers reached from the source by paths through that node; if it reaches the receivers, it must have reached the node. Conditioning on arrival at

${ }^{*}$ This work was supported in part by DARPA and the AFL under agreement F30602-98-2-0238 
a descendent, we can determine the probability of successful transmission to and beyond the given node. Efficient inference algorithms are given in [2] for loss, [15] for delay distributions, [9] for delay variances, and [3] for inferring the logical multicast tree topology itself. Extensions of these ideas to unicast (where multicast is replaced by a packet pair [5] or a packet stripe [10]) have also been proposed.

All of the algorithms based on the MINC methodology rely on the availability of complete information from the receivers. This poses a serious problem in their deployment. For example, one promising avenue of deployment is through the extension of RTCP, the RTP [20] control protocol, to provide extended loss reports [11]. By piggybacking MINC loss reports on a standard transport protocol, one can effectively co-opt regular applications and their traffic to form a lightweight impromptu measurement infrastructure that encompasses many host end-points. However, loss reports are typically transmitted unreliably. Furthermore, the RTP standard imposes a constraint on the bandwidth that can be used by RTCP packets. Thus, this deployment will result in the availability of only incomplete data sets for the purpose of network inference. The need to analyze incomplete data sets also arises in the extension of the MINC techniques to unicast as they rely on collecting data from subsets of receivers. Thus there is a need to modify the inferencing techniques to be able to handle incomplete data sets. Using loss as an example, the goal of this paper is to extend the techniques developed in [2] to handle incomplete data.

\subsection{Contribution}

In this paper we adapt the multicast inference techniques proposed in [2] to perform inference of internal network characteristics when data is missing from some of the receivers. The data for the inference comprises measured end-to-end loss of multicast probes sent from a source to a number of destinations but where only a subset of the destinations report their observations for each multicast probe. This is used to infer the loss characteristics of each logical link of the tree joining the source to the destinations, i.e., of the composite paths between its branch points.

A simple approach to manage the impact of missing data would be to restrict inference to subsets of probes and receivers for which complete data is available, then patch together such estimators to draw inference on the complete tree. There are three drawbacks with this approach: (i) unless the coverage is sufficiently rich, it is not possible to infer transmission probabilities for all links; (ii) unless the missing data distribution obeys certain conditions-known as Missing Completely at Random (MCAR)-such estimators are not consistent in that they remain biased even in the limit of infinitely many probes; and (iii) even under MCAR such estimators are not generally efficient, i.e., there can exist estimators with smaller variance.

For these reasons we follow a more direct approach. We extend the Maximum Likelihood (ML) formulation of [2] to include the occurrence of missing data. The link loss probabilities are then estimated by the Maximum Likelihood Estimator (MLE) arising from the corresponding likelihood function. In contrast to the results in [2], it is not generally possible to determine the MLE by simple root finding when data is missing. Instead, we use the Expectation Maximization (EM) algorithm [7] to generate an approximating sequence to the corresponding MLE. We now outline the remainder of the paper and the detailed contributions.

In Section 2 we set up models for the multicast tree, probe propagation, and report loss, and review some 
results for loss inference from complete data from [2]. We describe the model frameworks for missing data and give two examples: inference using unicast stripes [10], and inference using extended RTCP reports, as proposed in [1]. In Section 3 we set up the incomplete data likelihood function, and describe the EM algorithm and its application to the present model. We establish conditions required for convergence of the EM iterates to the MLE. We translate these into conditions on the measured data. If these conditions are not fulfilled, it is possible to pass instead to one or more related inference problems on subtrees for which the conditions are fulfilled. In Section 4 we tailor the EM algorithm to our specific problem and present an algorithm for use on measured data. Section 5 addresses conditions for identifiability of model parameters and relates these to topological properties of families of subtrees on which complete measurements can be made. Convergence of the MLE as the number of probes grows is investigated in Section 6; in particular we obtain explicit expressions for the asymptotic variance of the MLE for a class of simple models. A related expression for the convergence rate of the EM iterates is obtained in Section 7. The algorithm from Section 4 is evaluated in model-based simulation and using experimentally derived traces in Section 8. We conclude in Section 9. Details of most proofs are deferred to Section 10.

\subsection{Related work}

Several tools and methodologies exist for characterizing link-level behavior from end-to-end multicast measurements. However, most of these require complete data from all of the receivers in the multicast tree. These include the MINC methodologies for losses, [2], and for delay, [15, 9] and topology characteristics, [3]. These methodologies have been adapted to unicast through the transmission of packet pairs [5] or stripes [10] to pairs of receivers within a distribution tree. The data then consists of observations from pairs of receivers and can be interpreted as observations in which the data is missing from all but these pairs of receivers. The methodology presented in [10] treats the problem as separate problems corresponding to each pair of receivers and produces link estimates by averaging over all of the estimates produced from each of these receiver pair problems.

In [5], the authors introduce an additional link parameter, namely the conditional probability that the second packet within a pair is not lost given that the first packet is not lost. The authors then treat the outcomes of the each of the packets in a pair within the tree as unobserved data and use the EM algorithm to infer the link probabilities and conditional link probabilities. Due to the complexity of this task, they propose a heuristic for inferring these parameters. Because we rely on multicast, our task is simplified as we only have one set of link parameters to infer. Our solution methodology uses the EM algorithm to obtain a solution to the likelihood equation. Coates and Nowak have extended their EM-based, unicast-based techniques to infer delay statistics in [6].

Last, there exist several approaches that infer round trip link behavior. These include pathchar $[8,12]$ and the linear algebraic approach of [21]. The former infers loss, delay, and available link bandwidth whereas the latter infers round trip link delays. The former requires considerable time to converge. Both lose accuracy with asymmetric round trip paths. 


\section{Models for Probe and Report Transmission}

\subsection{Tree model}

Let $\mathcal{T}=(V, L)$ denote a logical multicast tree with nodes $V$ and links $L$. We identify one node, the root $\rho$, with the source of probes, and set of leaves $R \subset V$ with the set of receivers. We assume that the root has single child, denoted by 1 . If not, then we can treat separately the trees descended through each child of $\rho$, each one having this property. Each node $k$, apart from the root $\rho$, has a parent $f(k)$ such that $(f(k), k)$ is a link in $L$. We will sometimes refer to the link $(f(k), k)$ that terminates at $k$ simply as link $k$. Define recursively the ancestors of $k$ by $f^{n}(k)=f\left(f^{n-1}(k)\right)$ with $f^{0}(k)=k$. We say $j$ is descended from $k$, and write $j \prec k$, if $k=f^{n}(j)$ for some $n \in \mathbb{N}$. The set of children of $k$, namely $\{j \in V: f(j)=k\}$ is denoted by $d(k) . \mathcal{T}(k)=(V(k), L(k))$ will denote the subtree rooted at $k ; R(k)=R \cap V(k)$ is the set of receivers in $\mathcal{T}(k)$. Define $U=V \backslash\{\rho\}$.

\subsection{Packet loss model}

We assume a Bernoulli loss model in which probes are independent and each probe is successfully transmitted across link $k$ with probability $\alpha_{k}$. Thus the progress of each probe down the tree is described by an independent copy of a stochastic process $X=\left(X_{k}\right)_{k \in V}$ as follows. $X_{\rho}=1 . X_{k}=1$ if the probe reaches node $k \in V$ and 0 otherwise. If $X_{k}=0$, then $X_{j}=0, \forall j \prec k$. Otherwise, $P\left[X_{j}=1 \mid X_{f(j)}=1\right]=\alpha_{j}$ and $P\left[X_{j}=0 \mid X_{f(j)}=1\right]=1-\alpha_{j}$. We adopt the convention $\alpha_{\rho}=1$ and denote $\alpha=\left(\alpha_{i}\right)_{i \in V}$. $\mathrm{P}_{\alpha}$ will denote the distribution of $X$.

\subsection{Inference of link loss from complete data}

When a probe is sent down the tree from the root $\rho$, we cannot observe the whole process $X$. We assume that, at most, we know only the outcome $\left(X_{k}\right)_{k \in R} \in \Omega=\{0,1\}^{R}$ that indicates whether or not the probe reached each receiver. When the entire outcome for a probe is known (i.e. $X_{k}$ for all receivers $k$ ), we shall say that we have complete data from that probe. In [2] it was shown how the link probabilities can be determined from the the distribution of (complete) outcomes. We briefly review this.

Consider an experiment in which $n$ probes are dispatched from the root $\rho$. Each probe $i=1, \ldots, n$ gives rise to an independent realization $X^{(i)}$ of the probe process $X$. We call

$$
X_{\mathrm{cplt}}=\left(X_{k}^{(i)}\right)_{k \in R}^{i=1, \ldots, n}
$$

the complete data for the experiment. For each outcome $x \in \Omega$, let $n(x)$ denote the number of probes $i=1, \ldots n$ for which $X_{k}^{(i)}=x_{k}$ for all $k \in R$. Let

$$
p_{\alpha}(x)=\mathrm{P}_{\alpha}\left[X_{k}=x_{k}, \forall k \in R\right]
$$

denote the probability of an outcome $x \in \Omega$. The complete data log-likelihood to obtain the data $X_{\text {cplt }}=$ $\left(X^{(1)}, \ldots, X^{(n)}\right)$ can be written in terms of the $n(x)$ as

$$
\mathcal{L}_{c}(\alpha)=\log \mathrm{P}_{\alpha}\left[X_{\mathrm{cplt}}\right]=\sum_{x \in \Omega} n(x) \log p_{\alpha}(x) .
$$


We characterize the Maximum Likelihood Estimator (MLE) of $\alpha$, namely, $\arg \max _{\alpha} \mathcal{L}(\alpha)$, as follows. For $k \in V$, let $A_{k}$ be the probability that the probe reaches $k$. Thus $A_{k}=\prod_{j \succeq k} \alpha_{k}$, the product of the probabilities of successful transmission on each link between $k$ and the root $\rho$. For each $k \in U$ set

$$
\gamma_{k}=\mathrm{E}_{\alpha}\left[\vee_{j \in R(k)} X_{j}\right]
$$

i.e., $\gamma_{k}$ is the probability that a probe reaches at least one receiver descended from node $k$. Denote by $\widehat{\gamma}_{k}$ the corresponding empirical quantity, i.e., the proportion of the $n$ probes that reach at least one leaf descended from $k$ :

$$
\widehat{\gamma}_{k}=n^{-1} \sum_{i=1}^{n} \vee_{j \in R(k)} X_{j}^{(i)} .
$$

In what follows we consider $\alpha$ to lie in the open parameter set $\mathcal{A}=\left\{\alpha \mid \alpha_{k} \in(0,1), k \in U\right\}$. Some of the results of the following theorem also hold on subsets of the boundary of $\mathcal{A}$.

Theorem 1 ([2]) Assume $\alpha \in \mathcal{A}$.

(i) For each $k \in U$,

$$
\left(1-\gamma_{k} / A_{k}\right)=\prod_{j \in d(k)}\left(1-\gamma_{j} / A_{k}\right)
$$

with the convention that an empty product occurs when $k \in R$ is zero.

(ii) Let $\mathcal{G}=\left\{\left(\gamma_{k}\right)_{k \in U}: \gamma_{k}>0 \forall k ; \gamma_{k}<\sum_{j \in d(k)} \gamma_{j} \forall k \in U \backslash R\right\}$. For each $\gamma \in \mathcal{G}$ and $k \in U$, (6) has a unique solution $\mathcal{H}_{k}(\gamma)$ in the interval $\left(\gamma_{k}, 1\right)$.

(iii) When $\widehat{\gamma} \in \mathcal{G}$, the likelihood equation,

$$
\frac{\partial \mathcal{L}_{c}}{\partial \alpha_{k}}(\alpha)=0, \quad k \in U
$$

has as a unique solution

$$
\widehat{\alpha}_{k}=\mathcal{K}_{k}(\widehat{\gamma}):=\mathcal{H}_{k}(\widehat{\gamma}) / \mathcal{H}_{f(k)}(\widehat{\gamma}), k \in U .
$$

(iv) With probability one, for sufficiently large $n$, both $\widehat{\alpha}$ and the MLE of $\alpha$ lie in $\mathcal{A}$, and are hence equal.

(v) The parameters $\alpha$ are identifiable, i.e., $\mathrm{P}_{\alpha}=\mathrm{P}_{\alpha^{\prime}}$ for $\alpha, \alpha^{\prime} \in \mathcal{A}$ implies $\alpha=\alpha^{\prime}$.

It turns out that Theorem 1(iv) is weaker than required for the present paper. We now establish a stronger version that provides a test as to whether or not $\widehat{\alpha}_{k}$ is the MLE for finite $n$.

Theorem 2 Assume $\widehat{\gamma} \in \mathcal{G}$. If $\widehat{\alpha} \in \mathcal{A}$, then it is the MLE for $\alpha$.

Remark: Theorem 1(iv) establishes that for $n$ sufficiently large, the MLE lies in $\mathcal{A}$ and hence must be $\widehat{\alpha}$, the solution of the likelihood equation. Theorem 2 is more useful from the computational point of view; it says that provided $\widehat{\alpha}$ lies in $\mathcal{A}$, a condition that can be checked by inspection, it is the MLE, regardless of $n$.

As a consequence of the MLE property, $\widehat{\alpha}$ is consistent $(\widehat{\alpha} \stackrel{n \rightarrow \infty}{\longrightarrow} \alpha$ with probability 1$)$, and asymptotically normal $(\sqrt{n}(\widehat{\alpha}-\alpha)$ converges in distribution to a multivariate Gaussian random variable as $n \rightarrow \infty)$; see e.g. [19]. 


\subsection{Missing data model}

We now want to generalize the problem by admitting the possibility that some outcomes may not be completely known because the receiver variables are missing. Let $\left.T=\left(T_{k}^{(i)}\right)\right)_{k \in R}^{i=1, \ldots, n}$ denote the $n \times \# R$ matrix of missing data indicators, with $T_{k}^{(i)}$ taking the value 0 if the variable $X_{k}^{(i)}$ is missing, and $T_{k}^{(i)}=1$ if it is present. The set of observed data and missing data are thus, respectively,

$$
X_{\mathrm{obs}}=\left\{X_{k}^{(i)} \mid T_{k}^{(i)}=1\right\} \quad \text { and } \quad X_{\mathrm{mis}}=\left\{X_{k}^{(i)} \mid T_{k}^{(i)}=0\right\} .
$$

In this paper we assume that the missing data mechanism is ignorable in a sense we now describe; see [14] for further details. We treat $T$ as a random variable whose distribution is parameterized by some quantity $\theta . \mathrm{P}_{\theta}$ will denote the distribution of $T$ under $\theta$, and $P_{\alpha, \theta}$ the joint distribution of $X_{\text {cplt }}$ and $T$. We henceforth assume that the missing data is missing at random (MAR). This is the property that the distribution of the missing-data mechanism $T$ does not depend on the missing values $X_{\text {mis. }}$ More formally, we can write the MAR property as $\mathrm{P}_{\theta}\left[T \mid X_{\text {obs }}, X_{\text {mis }}\right]=\mathrm{P}_{\theta}\left[T \mid X_{\text {obs }}\right]$. As a consequence of MAR it can be shown that the joint distribution of the observed data and the missing-data mechanism enjoys the following factorization property:

$$
\mathrm{P}_{\theta, \alpha}\left[X_{\text {obs }}, T\right]=\mathrm{P}_{\theta}\left[T \mid X_{\text {obs }}\right] \mathrm{P}_{\alpha}\left[X_{\text {obs }}\right] .
$$

Assuming the parameters $(\alpha, \theta)$ to be distinct with product parameter space $\mathcal{A} \times \Theta,(10)$ says that the missing data mechanism is ignorable in that likelihood-based inference for $\alpha$ based on the joint likelihood $\mathrm{P}_{\theta, \alpha}\left[X_{\text {obs }}, T\right]$ are the same as those based upon $\mathrm{P}_{\alpha}\left[X_{\mathrm{obs}}\right]$. Thus for purposes of inferring $\alpha$, we may ignore the parameters $\theta$ of the missing data mechanism. A special case of MAR is data missing completely at random (MCAR). With MCAR the missingness probabilities do not depend on any data: $\mathrm{P}_{\theta}\left[T \mid X_{\text {obs }}\right]=$ $\mathrm{P}_{\theta}[T]$.

\subsection{Examples}

We describe two applications where data is missing and place them into the framework described above.

Inference using unicast data. In [10], the authors describe an approach to unicast based inference in which $n$ sets of packets, known as stripes, are transmitted by a source to all receiver pairs. The motivation is that within each stripe, packets are transmitted back-to-back, and so their loss behavior on common links should be highly correlated. With perfect correlations (i.e. both packets being either transmitted or lost on a common link) the stripe has the same behavior as a notional multicast packet that follows the same route and is subject to the same loss.

We can put each receiver pair in correspondence with a missing data indicator as follows. $T^{(i)}=$ $\left(T_{k}^{(i)}\right)_{k \in R}$ identifies the pair of receivers corresponding to the $i$-th stripe, i.e., $T_{j}^{(i)}=T_{k}^{(i)}=1, T_{l}^{(i)}=0$, $l \in R, l \neq j, k$ if the pair of receivers is $j, k \in R, j \neq k$. Thus missingness of data from probe $i$ at receiver $\ell$ occurs because $\ell$ is not a member of the pair of receiver nodes selected for the probe. 
If the receiver pairs are chosen independently from stripe to stripe using the same distribution, then $T=\left(T^{(i)}\right)_{i=1}^{n}$ is a sequence of IID random variables. Thus $T$ has the following distribution,

$$
\mathrm{P}[T=t]=\prod_{i=1}^{n} \mathrm{P}\left[T^{(i)}=t^{(i)}\right], \quad \forall t \in\{0,1\}^{\# R}
$$

where

$$
\mathrm{P}\left[T^{(i)}=t\right]=\sum_{j, k \in R ; j \neq k} \mathbf{1}\left\{t_{j}=1\right\} \mathbf{1}\left\{t_{k}=1\right\} \prod_{l \in R \backslash\{j, k\}} \mathbf{1}\left\{t_{l}=0\right\} p_{j, k}, \quad \forall t \in\{0,1\}^{\# R}
$$

Here $p_{j, k}$ is the probability that the pair of receivers $j$ and $k$ is chosen. If we further assume that $T$ is independent of $X$, then the data is MCAR.

Another variation has the sender cycle through the pairs in a round robin fashion. Let $\pi: R^{2} \backslash\{(j, j)$ : $j \in R\} \rightarrow\{1, \ldots, m\}$ be a one-to-one mapping where $\pi(j, k)$ is the position in the round robin schedule where a probe is sent to receiver pair $j$ and $k$ and $m=\# R \times(\# R-1)$. The joint probability distribution for $T$ is given by (11) with

$$
\mathrm{P}\left[T^{(m i+d)}=t\right]=\sum_{j, k \in R ; j \neq k} \mathbf{1}\{\pi(j, k)=d\} \mathbf{1}\left\{t_{j}=1\right\} \mathbf{1}\left\{t_{k}=1\right\} \prod_{l \in R \backslash\{j, k\}} \mathbf{1}\left\{t_{l}=0\right\},
$$

for all $t \in\{0,1\}^{\# R}, i \geq 0,1 \leq d \leq m$.

Inference using RTP/RTCP. The Reliable Transport Protocol (RTP) [20] is a protocol for the transfer of data from a single sender to one or more receivers. Associated with it is a control protocol RTCP that allows receivers to broadcast loss behavior to each other and to a third party. Typically, the observations are batched and each batch is broadcast as a single report. The third party can collect the observations and apply inference methodologies to them. However, these reports are typically not transmitted reliably. Consequently, the data collector must deal with missing information.

In the current implementation of RTCP, receivers broadcast only average loss rates. Extensions to the protocol, proposed in [1], enable receivers to report on the reception of individual packets. However, due to the constraints imposed on reporting volumes by RTCP, it may not be possible to report on every packet. The omission of certain reports to fulfill this constraint is thus an additional source of missingness.

We propose a simple model for this scenario. Consider receiver $j \in R$ that collects loss observations and sends them to a data collector. Let $\left\{\left\{A_{i, j}\right\}_{i=1}^{\infty}\right\}_{j \in R}$ be a set of random variables where $A_{i, j}$ is the number of observations placed in the $i$-th report by the $j$-th receiver. Let $\left\{\left\{C_{j}^{(k)}\right\}_{k=1}^{\infty}\right\}_{j \in R}$ be indicator random variables that represent the outcome of the transmission of the $k$-th loss report by receiver $j$ to the data collector; it is received by the collector if $C_{j}^{(k)}=1$ and lost otherwise. Define $\pi(i, j)=\min \{l$ : $\left.\sum_{k=1}^{l-1} A_{k, j}<i \leq \sum_{k=1}^{l} A_{k, j}\right\}$, i.e., $\pi(i, j)$ identifies which report the $i$-th observation from receiver $j$ is placed in. Let $\left\{\left\{S_{j}^{(i)}\right\}_{i=1}^{\infty}\right\}_{j \in R}$ be a set of indicator random variables that represent whether probe $i$ was actually selected for reporting from receiver $j$; it is selected if $S_{j}^{(i)}=1$. Then the missing data indicator $T_{j}^{(i)}, i=1, \ldots, n, j \in R$ can be expressed as $T_{j}^{(i)}=S_{j}^{(i)} C_{j}^{(\pi(i, j))}$. 
Under strong simplifying assumptions, namely that the random variables $A, S$ and $C$ are independent of $X$, the model is MCAR. However we can posit a situation in which independence may not hold in practice. Suppose the collector lies at a node $k$ in the multicast tree. Then the path for reports from receivers in $R \backslash R(k)$ to $k$ intersects with the paths of probe packets from $\rho$ to receivers in $R(k)$. Thus we may expect the missingness variables $\left\{T_{j}^{(i)}: j \in R \backslash R(k)\right\}$ to be correlated with the receiver state $\left\{X_{j}: j \in R(k)\right\}$. This is precisely the type of model allowed when data is MAR.

\subsection{Approaches to the problem of missing data}

It is tempting to reduce the problem of inference with missing data to a composite of known inferences by performing inference using subsets of probes for which reports reached leaf descendents of a given node. A simple approach to manage the impact of missing data is to restrict inference to subsets of probes and receivers for which complete data is available, then patch together such estimators to draw inference on the complete tree. A minimal way to do this would be to use only probes for which reports were received from all receivers. A more sophisticated approach is the following:

(a) For each $k \in R$, estimate $\widehat{A}_{k}=\widehat{\gamma}_{k}$ by the fraction of observed reports indicating probe reception.

(b) For each $k \in U \backslash R$ let $\mathcal{R}_{k}$ denote the set of subsets of $R(k)$ in which each member is the descendant of a different child of $k$. For each $r \in \mathcal{R}_{k}$, use only probes with reports received from all $j \in r$ to form the fractions $\widehat{\gamma}_{k}(r)$ and $\left\{\widehat{\gamma}_{j}(r)\right\}_{j \in r}$. Estimate $\widehat{A}_{k}=\left(\sum_{r \in \mathcal{R}_{k}} \mathcal{H}_{k}(\widehat{\gamma}(r)) / \# \mathcal{R}_{k}\right.$, i.e., averaging over the $r \in \mathcal{R}_{k}$.

(c) Estimate link transmission probabilities $\widehat{\alpha}_{k}=\widehat{A}_{k} / \widehat{A}_{f(k)}$.

However, such "patchwork" approaches have three pitfalls:

(i) Unless the coverage is sufficiently rich, it is not possible to infer transmission probabilities for all links. If not all nodes are branch points of some "complete data" subtree, it follows from one of our later results that one cannot infer the transmission probability for the link that terminates at that node. In the minimal case, there may be no probes for which reports are received from all probes.

(ii) Such estimators are not consistent unless data is MCAR; we illustrate with an example in Section 3.1. Furthermore, checking whether a given data set is consistent with the MCAR property may be a complex task since the number of consistency conditions that would have to be checked grows exponentially with the number of leaves in the tree.

(iii) Even under MCAR such estimators are not generally efficient, i.e., there can exist estimators with smaller variance.

For these reasons we instead extend the previous ML approach to cover the missing data case directly: under general conditions ML-estimators are consistent and efficient. This is the subject of the next section. 


\section{Estimation of Link Loss Rates with Incomplete Data}

In this section we present the likelihood function $\mathcal{L}$ for the incomplete data. Determination of the corresponding ML estimator for the link probabilities turns out to be significantly more complex that in the complete data case. We turn to a standard iterative method, the EM algorithm, to derive an approximating sequence to the incomplete data MLE.

\subsection{Description of incomplete data and the likelihood function}

The corresponding incomplete data likelihood function is the marginal distribution function of the observed data; formally we write this as $\int P_{\alpha}\left[X_{\mathrm{obs}}, X_{\mathrm{mis}}\right] d X_{\mathrm{mis}}$. We now obtain an explicit expression. In order to represent both missing and observed data in a compact form, we extend the set of outcomes to the set $\Omega^{*}=\{0,1, \mathrm{u}\}^{R}$, where $\mathrm{u}$ is used to denote that a given receiver datum is missing. $\mathrm{u}^{*}=(\mathrm{u}, \ldots, \mathrm{u}) \in \Omega^{*}$ will denote the outcome in which data is missing from all receivers. Let $t \in\{0,1\}^{R}$ denote the generic vector of missing data indicator variables. With each such $t$ and $x \in \Omega$ we then associate an element $x(t)$ of $\Omega^{*}$ through

$$
x_{k}(t)=\left\{\begin{array}{ll}
\mathrm{u} & \text { if } t_{k}=0 \\
x_{k} & \text { otherwise }
\end{array}, k \in R\right.
$$

An inverse of the above map associates with $x^{*} \in \Omega^{*}$ its missing data indicator $t\left(x^{*}\right)$ by

$$
t_{k}\left(x^{*}\right)=\left\{\begin{array}{ll}
0 & \text { if } x_{k}^{*}=u \\
1 & \text { otherwise }
\end{array}, k \in R .\right.
$$

The set of complete outcomes $x$ that can give rise to an incomplete outcome $x^{*}$ is the set

$$
\Omega\left(x^{*}\right)=\left\{x \in \Omega \mid x_{k}^{*}=x_{k} \Leftrightarrow t_{k}\left(x^{*}\right)=1\right\}, \quad \text { and conversely } \Omega^{*}(x)=\left\{x^{*} \in \Omega^{*} \mid x \in \Omega\left(x^{*}\right)\right\}
$$

is the set of complete outcomes $x^{*}$ that can be obtained from a complete outcome $x$. The equivalent conditions $x \in \Omega\left(x^{*}\right)$ and $x^{*} \in \Omega^{*}(x)$ can be rewritten as $x\left(t\left(x^{*}\right)\right)=x^{*}$.

The probability to record an incomplete outcome $X^{(i)}\left(T^{(i)}\right)=x^{*}$ is denoted

$$
q_{\alpha, \theta}\left(x^{*}\right)=\mathrm{P}_{\alpha, \theta}\left[X^{(i)}\left(T^{(i)}\right)=x^{*}\right] .
$$

Now $\left\{X^{(i)}\left(T^{(i)}\right)=x^{*}\right\}=\left\{X^{(i)} \in \Omega\left(x^{*}\right)\right\} \cap\left\{T^{(i)}=t\left(x^{*}\right)\right\}$. Using the MAR property (10) we factorize

$$
q_{\alpha, \theta}\left(x^{*}\right)=p_{\alpha}^{*}\left(x^{*}\right) \theta\left(x^{*}\right)
$$

where

$$
p_{\alpha}^{*}\left(x^{*}\right)=\mathrm{P}_{\alpha}\left[X^{(i)} \in \Omega\left(x^{*}\right)\right]=\sum_{x \in \Omega\left(x^{*}\right)} p_{\alpha}(x) \quad \text { and } \quad \theta\left(x^{*}\right)=\mathrm{P}_{\theta}\left[T^{(i)}=t\left(x^{*}\right) \mid X^{(i)} \in \Omega\left(x^{*}\right)\right] .
$$

Without loss of generality we have taken the missingness probabilities themselves as parameters $\theta$. Note that by the MAR property, for any $x \in \Omega\left(x^{*}\right), \mathrm{P}_{\theta}\left[T^{(i)}=t\left(x^{*}\right) \mid X^{(i)} \in \Omega\left(x^{*}\right)\right]=\mathrm{P}_{\theta}\left[T^{(i)}=t\left(x^{*}\right) \mid X^{(i)}=x\right]$. Since $\left\{t\left(x^{*}\right) \mid x^{*} \in \Omega^{*}(x)\right\}=\{0,1\}^{R}$, the conditional probabilities $\theta$ satisfy

$$
\sum_{x^{*} \in \Omega^{*}(x)} \theta\left(x^{*}\right)=1, \quad \forall x \in \Omega .
$$


Now let $m\left(x^{*}\right)$ denote the number of probes $i=1, \ldots, n$ for which $X^{(i)}\left(T^{(i)}\right)=x^{*}$. Due to the factorization property (10), the log-likelihood function $\log \prod_{i=1}^{n} q_{\alpha, \theta}\left(X^{(i)}\left(T^{(i)}\right)\right)$ can be written as a sum of

$$
\mathcal{L}(\alpha)=\sum_{x^{*} \in \Omega^{*}} m\left(x^{*}\right) \log p_{\alpha}^{*}\left(x^{*}\right)
$$

with a term that is independent of $\alpha$. Thus, for the purposes of obtaining an ML estimate of $\alpha$, we need only consider $\mathcal{L}(\alpha)$. We refer to $\mathcal{L}$ as the incomplete data likelihood function. Note that the term in $m\left(\mathrm{u}^{*}\right)$ makes no contribution to $\mathcal{L}$ since $\Omega\left(\mathrm{u}^{*}\right)=\Omega$ and hence $p_{\alpha}^{*}\left(\mathrm{u}^{*}\right)=1$. Hence the sum in (20) can be restricted to $\Omega_{0}^{*}=\Omega^{*} \backslash\left\{\mathrm{u}^{*}\right\}$.

Example: 2 leaf tree with MAR data. We now give an example to show how the complete data MLE, applied to those probes for which complete data is available, generally produces an inconsistent estimate of the link probabilities in the MAR case. Consider a two leaf tree where data is MAR from the right leaf; the probability of missingness thus depends on the data observed at the left leaf. The leaf probabilities obey:

$$
\begin{array}{rlr}
q(11)=\alpha_{1} \alpha_{2} \alpha_{3} \theta(11), & q(10)=\alpha_{1} \alpha_{2} \bar{\alpha}_{3} \theta(10), \quad q(01)=\alpha_{1} \bar{\alpha}_{2} \alpha_{3} \theta(01), \\
q(00)=\left(\bar{\alpha}_{1}+\alpha_{1} \bar{\alpha}_{2} \bar{\alpha}_{3}\right) \theta(00), & q(1 \mathrm{u})=\alpha_{1} \alpha_{2} \theta(1 \mathrm{u}), \quad q(0 \mathrm{u})=\left(1-\alpha_{1} \alpha_{2}\right) \theta(1 \mathrm{u})
\end{array}
$$

Using the four instances of (19), namely,

$$
\begin{aligned}
& x=11: \theta(11)+\theta(1 \mathrm{u})=1 ; \quad x=10: \theta(10)+\theta(1 \mathrm{u})=1 ; \\
& x=01: \theta(01)+\theta(0 \mathrm{u})=1 ; \quad x=00: \theta(00)+\theta(0 \mathrm{u})=1 ;
\end{aligned}
$$

(21) reduces to

$$
\begin{aligned}
q(11)=\alpha_{1} \alpha_{2} \alpha_{3} \theta(11), & q(10)=\alpha_{1} \alpha_{2} \bar{\alpha}_{3} \theta(11), \quad q(01)=\alpha_{1} \bar{\alpha}_{2} \alpha_{3} \theta(01), \\
q(00)=\left(\bar{\alpha}_{1}+\alpha_{1} \bar{\alpha}_{2} \bar{\alpha}_{3}\right) \theta(01), & q(1 \mathrm{u})=\alpha_{1} \alpha_{2} \bar{\theta}(11), \quad q(0 \mathrm{u})=\left(1-\alpha_{1} \alpha_{2}\right) \bar{\theta}(01)
\end{aligned}
$$

Now, the complete data MLE based on the corresponding complete data empirical probabilities $\widehat{q}(11), \widehat{q}(10), \widehat{q}(01), \widehat{q}(00)$ is

$$
\widehat{\alpha}_{1}=\frac{(\widehat{q}(11)+\widehat{q}(10))(\widehat{q}(11)+\widehat{q}(01))}{\widehat{q}(11)(\widehat{q}(11)+\widehat{q}(10)+\widehat{q}(01)+\widehat{q}(00))}, \quad \widehat{\alpha}_{2}=\frac{\widehat{q}(11)}{\widehat{q}(11)+\widehat{q}(01)}, \quad \widehat{\alpha}_{3}=\frac{\widehat{q}(11)}{\widehat{q}(11)+\widehat{q}(10)}
$$

In the MCAR case, all $\theta\left(x^{*}\right)$ appearing in (25) would be equal, and substituting $q$ for $\widehat{q}$ in (27) one recovers $\alpha_{1}, \alpha_{2}, \alpha_{3}$ : the estimator is consistent. But in the general MAR case one obtains only

$$
\frac{\alpha_{1}\left(\alpha_{2} \theta(11)+\bar{\alpha}_{2} \theta(01)\right)}{\alpha_{1} \alpha_{2} \theta(11)+\left(1-\alpha_{1} \alpha_{2}\right) \theta(01)} \neq \alpha_{1}, \quad \frac{\alpha_{2} \theta(11)}{\alpha_{2} \theta(11)+\bar{\alpha}_{2} \theta(01)} \neq \alpha_{2}, \quad \alpha_{3},
$$

i.e. only estimation of $\alpha_{3}$ is consistent. 


\subsection{Application of the EM algorithm}

We can in principle estimate the link probabilities $\alpha$ by the incomplete data MLE $\check{\alpha}=\arg \max _{\alpha} \mathcal{L}(\alpha)$ in (20) calculated from the counts of incomplete outcomes $\boldsymbol{m}=\left\{m\left(x^{*}\right): x^{*} \in \Omega_{0}^{*}\right\}$. However, we have been unable to obtain a direct solution to the incomplete-data likelihood equation. Instead, we employ a standard statistical method,the Expectation-Maximization (EM) algorithm [7], to obtain an iterative approximation $\widehat{\alpha}^{(\ell)}, \ell=0,1, \ldots$ to a stationary value of the incomplete data likelihood. The algorithm comprises the following steps:

(i) Initialization. Pick some initial link probabilities $\alpha^{(0)}$. This could be done, e.g., by setting $\widehat{\alpha}^{(0)}=\widehat{\alpha}$, the complete data MLE determined from the counts of complete outcomes $\boldsymbol{m}$ if these are non-zero. When complete data is not available, we can use the fact (see the proof of Theorem 5 in [2]) that $\gamma_{k}=$ $A_{k}+O\left(\|\bar{\alpha}\|^{2}\right)$ to approximate $\alpha_{k}=A_{k} / A_{f(k)} \approx \gamma_{k} / \gamma_{f(k)}=\left(1-\bar{\gamma}_{k}\right) /\left(1-\bar{\gamma}_{f(k)}\right) \approx 1+\gamma_{k}-\gamma_{f(k)}$. This suggests the initial value

$$
\widehat{\alpha}_{k}^{(0)}=1+\widehat{\gamma}_{k}-\widehat{\gamma}_{f(k)}
$$

(ii) Expectation. For each $\widehat{\alpha}^{(\ell)}$ find the conditional expectation of the complete log-likelihood given the incomplete data $Q\left(\alpha^{\prime}, \widehat{\alpha}^{(\ell)}\right)=\mathrm{E}_{\widehat{\alpha}^{(\ell)}}\left[\mathcal{L}_{c}\left(\alpha^{\prime}\right) \mid \boldsymbol{m}\right]$.

(iii) Maximization. Find the maximizer of the condition expectation: $\widehat{\alpha}^{(\ell+1)}=\arg \max _{\alpha^{\prime}} Q\left(\alpha^{\prime}, \widehat{\alpha}^{(\ell)}\right)$

(iv) Iteration. Iterate steps (ii) and (iii) until some termination criterion is satisfied.

For $k \in V$, define the conditional probabilities for a probe to reach $R(k)$ as

$$
\widehat{\gamma}_{k, \alpha}=\mathrm{E}_{\alpha}\left[\vee_{j \in R(k)} X_{k} \mid \boldsymbol{m}\right] .
$$

For notational convenience we write the conditional probability $\widehat{\gamma}_{k, \widehat{\alpha}^{(\ell)}}$ derived from the iterate $\widehat{\alpha}^{(\ell)}$ as $\widehat{\gamma}_{k}^{(\ell)}$.

Theorem 3 Assume $\widehat{\gamma}^{(\ell)} \in \mathcal{G}$. Then

$$
\alpha_{k}^{(\ell+1)}=\mathcal{K}_{k}\left(\widehat{\gamma}^{(\ell)}\right), k \in U
$$

provided that $\mathcal{K}\left(\widehat{\gamma}^{(\ell)}\right)$ lies in $\mathcal{A}$.

We now investigate the question of convergence of the iterates $\widehat{\alpha}^{(\ell)}$. Whereas the complete data likelihood function can be shown to derive from a standard exponential family (see the proof of Theorem 2), the incomplete data likelihood function derives only from a curved exponential family. Thus we cannot use results based on standard exponential families (see e.g. [22]) alone to conclude convergence of $\widehat{\alpha}^{(\ell)}$ to $\check{\alpha}$. We now establish conditions under which the sequence exists in $\mathcal{A}$ and converges to the MLE for the incomplete data problem.

Theorem 4 Assume $\widehat{\gamma}^{(\ell)} \in \mathcal{G}$ and $\mathcal{K}\left(\widehat{\gamma}^{(\ell)}\right) \in \mathcal{A}$ for all $\ell$.

(i) $\mathcal{L}\left(\widehat{\alpha}^{(\ell)}\right)$ converges to some limit $L$. 
(ii) If $\{\alpha \in \mathcal{A} \mid \mathcal{L}(\alpha)=L\}$ is discrete, $\widehat{\alpha}^{(\ell)}$ converges to some $\alpha^{*}$ that is a stationary point $\mathcal{L}$, i.e. $\frac{\partial \mathcal{L}}{\partial \alpha}\left(\alpha^{*}\right)=0$.

(iii) If $\mathcal{L}$ is unimodal, $\widehat{\alpha}^{(\ell)}$ converges to the incomplete data MLE $\check{\alpha}$.

\subsection{Calculation of the EM iterates}

An algorithm for the calculating $\mathcal{K}_{k}(\gamma)$ for a given $\gamma \in \mathcal{G}$ has been detailed in [2]. It remains then to provide an algorithm for the calculation of the $\widehat{\gamma}_{\alpha}$. Let $n_{0}=n-m^{*}\left(\mathbf{u}^{*}\right)$ denote the number of probes for which the data is not entirely missing. Observe that

$$
\widehat{\gamma}_{k, \alpha}=\sum_{x^{*} \in \Omega_{0}^{*}} \frac{m\left(x^{*}\right)}{n_{0}} \widehat{\gamma}_{k, \alpha}\left(x^{*}\right)
$$

where

$$
\widehat{\gamma}_{k, \alpha}\left(x^{*}\right)=\mathrm{E}_{\alpha}\left[\vee_{j \in R(k)} X_{k} \mid X^{*}=x^{*}\right] .
$$

Let $R\left(k, x^{*}\right)=\left\{j \in R(k) \mid t_{j}\left(x^{*}\right)=1\right\}$ denote the receivers descended from $k$ from which data is observable. Let $h\left(k, x^{*}\right)$ denote the closest ancestor $h$ of $k$ for which a packet has been observed to reach at least one descendant leaf, i.e.,

$$
h\left(k, x^{*}\right)=\inf _{j \succeq k}\left\{j: y_{j}^{*}=1\right\},
$$

where $y_{k}^{*}=\vee_{j \in R(k)}^{*} x_{j}^{*}$. When $k \prec j$, let $d(j, k)$ denote that child of $j$ that is an ancestor (or possibly equal to) $k$, i.e., $d(j, k)=\{i \in d(j): i \succeq k\}$.

Theorem 5 When $y_{k}^{*}=1, \widehat{\gamma}_{k, \alpha}\left(x^{*}\right)=1$. When $y_{k}^{*}<1$,

$$
\widehat{\gamma}_{k, \alpha}\left(x^{*}\right)=\frac{c_{k}-b_{k}}{c_{d(h, k)}} \prod_{k \prec i \preceq d(k, h)}\left\{\begin{array}{c}
\alpha_{i} \prod_{j \in d(i) \backslash d(i, k)} c_{j} \\
j \in
\end{array}\right.
$$

where $h=h\left(k, x^{*}\right)$, and for $k \preceq i \prec h$,

$$
b_{k}=\mathrm{P}_{\alpha}\left[\vee_{j \in R(k)} X_{j}=0 \mid X_{f(k)}=1\right], \quad c_{k}=\left\{\begin{array}{l}
1, \quad \text { if } R\left(k, x^{*}\right)=\emptyset \\
\mathrm{P}_{\alpha}\left[\mathrm{\vee}_{j \in R\left(k, x^{*}\right)} X_{j}=0 \mid X_{f(k)}=1\right] \\
\text { otherwise }
\end{array}\right.
$$

Remark: it was found in [2] that the problem of determining the $\alpha$ for a tree with complete data factors into the problem of solving a set of depth two tree inference problems, one for each node $k \in V \backslash R$. For each leaf $k$ one constructs the logical tree with root $\rho$ having single child $k$, and $d(k)$ leaf-children. Furthermore, for a general tree, the problem could be mapped onto that for a binary tree by the insertion of lossless links. However, this method cannot be applied when there is missing data. This is because the form (35) for $\widehat{\gamma}_{k, \alpha}^{(\ell)}$ includes variables from receivers other than those descended from $k$. 


\subsection{Topology and data conditions}

Theorems 3 and 4 required the iterators $\widehat{\gamma}^{(\ell)}$ to lie in the domain $\mathcal{G}$. In this section we specify conditions on the data in order for these requirements to hold. In some cases where the conditions do not hold, it is possible adjust the problem by passing to one or more subtrees of the original tree, for which the conditions do hold. The requirements for Theorems 3 and 4 are then fulfilled: see Lemma 1 below. The conditions describes here are similar to those applied in the case of complete data in [2].

Non-identifiable subtrees. Order the elements of the set $\{0,1, \mathrm{u}\}$ as $\mathrm{u}<0<1$ and extend the usual maximum operator $\vee$ on $\{0,1\}$ to an operation $V^{*}$ on $\{0,1, u\}$, respecting the order in an obvious manner. For a given realization $(X, T)$ of the single probe and missing data process, define the quantities

$$
Y_{k}^{*}=\vee_{j \in R(k)}^{*} X_{j}(T)
$$

i.e., the extended maximum of $X_{j}(T)$ over all receivers $j$ descended from $k . Y_{k}^{*}$ takes the value $\mathrm{u}$ if all data from $R(k)$ on a given probe is missing, 1 if a probe was observed to reach at least one receiver in $R(k)$, and 0 otherwise. We first eliminate from consideration subtrees on which no data is missing but whose leaves

were reached by no probes. For $k \in V$, let $D_{k}$ denote the event that for some probe $i, X_{j}^{(i)}\left(T^{(i)}\right) \neq 0$ for some $j \in R(k)$. We will assume

$$
D_{k} \text { occurs for all } k \in V
$$

If (38) does not hold, the following procedure reduces the inference problem to one for which it does. If $D_{k}$ fails, we remove from further consideration the subtree $\mathcal{T}(k)$ rooted at $k$. If this pruning leaves the parent $f(k)$ with only one offspring $j$, the remaining tree is no longer a logical multicast tree. To make it so we remove the link $(f(k), j)$ and identify the nodes $j$ and $f(k)$. The consequence is that we will only able to identify the characteristics of the composite link joining $j$ to $f^{2}(j)$ of the original tree. Performing these operations for all $k$ at which $D_{k}$ fails, we obtain a tree for which (38) holds.

In general, it is not possible to attribute a transmission probability, even of zero, to individual links in $\mathcal{T}(k)$, since we cannot distinguish the link or links with zero transmission rate. An exception to this is when $D_{k}$ fails for a leaf node $k$, but $D_{f(k)}$ holds at the parent node $f(k)$. In this case we may estimate $\widehat{\alpha}_{k}=0$. Except in this case, we flag all $\left\{\alpha_{j}: j \preceq k\right\}$ as unknown.

Links with perfect transmission. Let $D_{k}^{\prime}$ denote the complement of the event $\left\{X_{j}^{(i)}\left(T^{(i)}\right)=1, \forall j \in\right.$ $R(k), i=1, \ldots, n\}$. When $D_{k}^{\prime}$ fails, lossless transmission is reported for all probes to all receivers in $R(k)$. The effect is to position $\mathcal{K}\left(\widehat{\gamma}_{\alpha}\right)$ on the boundary of $\mathcal{A}$, since it follows that $\mathcal{K}_{j}\left(\widehat{\gamma}_{\alpha}\right)=1$ for all $j \in R(k)$. Although this is not a problem for computation, it takes us out of the domain of application of Theorems 2 , 3 and 4 . The formal application of these results requires that

$$
D_{k}^{\prime} \text { holds for all } k \in V \text {. }
$$

If (39) does not hold, the following procedure reduces the inference problem to a set of one or more for which it does. When $D_{k}^{\prime}$ fails, we set $\widehat{\alpha}_{j}=1$ for all nodes $j \in V(k)$, and omit these nodes from further 
consideration. We then spawn a set of separate inference problems by forming the set of subtrees not containing $k$ that are rooted at ancestors of $k$. This is the set of trees $\{\mathcal{T}(j, \ell) \mid j \succ k ; \ell \in d(j), \ell \nsucc k\}$, where $\mathcal{T}(j, \ell)$ has vertices $\{j\} \cup V(\ell)$ and links $\{(j, \ell)\} \cup L(\ell)$.

Model Conformance. We also need a condition to ensure that estimated quantities $\widehat{\gamma}$ lie in $\mathcal{G}$. Let $D_{k}^{\prime \prime}$ be the event that $k$ has children $j, \ell \in d(k)$ such that $X_{j}^{(i)}\left(T^{(i)}\right)=1$ and $X_{\ell}^{(i)}\left(T^{(i)}\right) \neq 0$. Without this condition, probe losses on different subtrees descended from $k$, conditional on the probe having reached $k$, are correlated. This is because each probe is observed on no more than one such subtree. Henceforth we assume

$$
D_{k}^{\prime \prime} \text { holds for all any } k \in V \backslash R \text {. }
$$

If $D_{k}^{\prime \prime}$ fails, we adjust the tree by removing the link $(f(k), k)$ from the tree and identifying its endpoints $k$ and $f(k)$. In the original tree, we will only be able to identify the characteristics of the composite links joining $f(k)$ to the children $d(k)$. The procedure is iterated if necessary until (40) holds.

Conditions (38) and (40) enable us to fulfill some assumptions in Theorems 3 and 4. We will henceforth assume that they hold.

Lemma 1 When (38) and (40) hold, $\widehat{\gamma}_{\alpha} \in \mathcal{G}$ for any $\alpha \in \mathcal{A}$.

\subsection{Example: the two-receiver tree}

In the simplest case we can establish unimodality of $\mathcal{L}_{c}$ directly, and thus conclude convergence of the EM iterates to the incomplete data MLE. Consider the two receiver tree with root $\rho$ having a single child 1 whose children are the leaf nodes 2 and 3 . In the two receiver tree, we enumerate $\Omega=\{11,01,10,00\}$ and $\Omega_{0}^{*}=\{11,01,10,00,1 \mathrm{u}, \mathrm{u} 1,0 \mathrm{u}, \mathrm{u} 0\}$. It is not difficult to determine that the $\widehat{\gamma}_{k, \alpha}\left(x^{*}\right)$ are as given by the following table:

These yield

\begin{tabular}{l|lll}
$x^{*}$ & $\widehat{\gamma}_{1, \alpha}\left(x^{*}\right)$ & $\widehat{\gamma}_{2, \alpha}\left(x^{*}\right)$ & $\widehat{\gamma}_{3, \alpha}\left(x^{*}\right)$ \\
\hline 11 & 1 & 1 & 1 \\
10 & 1 & 1 & 0 \\
01 & 1 & 0 & 1 \\
00 & 0 & 0 & 0 \\
$1 \mathrm{u}$ & 1 & 1 & $\alpha_{3}$ \\
$\mathrm{u} 1$ & 1 & $\alpha_{2}$ & 1 \\
$0 \mathrm{u}$ & $\frac{\alpha_{1} \bar{\alpha}_{2} \alpha_{3}}{\bar{\alpha}_{1}+\alpha_{1} \bar{\alpha}_{2}}$ & 0 & $\frac{\alpha_{1} \bar{\alpha}_{2} \alpha_{3}}{\bar{\alpha}_{1}+\alpha_{1} \bar{\alpha}_{2}}$ \\
u0 & $\frac{\alpha_{1} \alpha_{2} \alpha_{3}}{\bar{\alpha}_{1}+\alpha_{1} \bar{\alpha}_{3}}$ & $\frac{\alpha_{1} \alpha_{2} \bar{\alpha}_{3}}{\bar{\alpha}_{1}+\alpha_{1} \bar{\alpha}_{3}}$ & 0
\end{tabular}

$$
\begin{aligned}
& n_{0} \widehat{\gamma}_{2, \alpha}=m(11)+m(10)+m(1 \mathbf{u})+m(\mathbf{u} 1) \alpha_{2}+m(\mathbf{u} 0) \frac{\alpha_{1} \alpha_{2} \bar{\alpha}_{3}}{\bar{\alpha}_{1}+\alpha_{1} \bar{\alpha}_{3}} \\
& n_{0} \widehat{\gamma}_{3, \alpha}=m(11)+m(01)+m(\mathbf{u} 1)+m(1 \mathbf{u}) \alpha_{3}+m(0 \mathbf{u}) \frac{\alpha_{1} \bar{\alpha}_{2} \alpha_{3}}{\bar{\alpha}_{1}+\alpha_{1} \bar{\alpha}_{2}} \\
& n_{0}\left(\widehat{\gamma}_{2, \alpha}+\widehat{\gamma}_{3, \alpha}-\widehat{\gamma}_{1, \alpha}\right)=m(11)+m(1 \mathbf{u}) \alpha_{3}+m(\mathbf{u} 1) \alpha_{2}
\end{aligned}
$$

The EM iterates $\left(\mathcal{K}_{1}\left(\widehat{\gamma}_{\alpha}\right), \mathcal{K}_{2}\left(\widehat{\gamma}_{\alpha}\right), \mathcal{K}_{3}\left(\widehat{\gamma}_{\alpha}\right)\right)$ of $\left(\alpha_{1}, \alpha_{2}, \alpha_{3}\right)$ are then

$$
\mathcal{K}_{1}\left(\widehat{\gamma}_{\alpha}\right)=\frac{\widehat{\gamma}_{2, \alpha} \widehat{\gamma}_{3, \alpha}}{\widehat{\gamma}_{2, \alpha}+\widehat{\gamma}_{3, \alpha}-\widehat{\gamma}_{1, \alpha}}, \quad \mathcal{K}_{2}\left(\widehat{\gamma}_{\alpha}\right)=\frac{\widehat{\gamma}_{2, \alpha}+\widehat{\gamma}_{3, \alpha}-\widehat{\gamma}_{1, \alpha}}{\widehat{\gamma}_{3, \alpha}}, \quad \mathcal{K}_{3}\left(\widehat{\gamma}_{\alpha}\right)=\frac{\widehat{\gamma}_{2, \alpha}+\widehat{\gamma}_{3, \alpha}-\widehat{\gamma}_{1, \alpha}}{\widehat{\gamma}_{2, \alpha}}
$$


Theorem 6 In the two-receiver tree, the incomplete data likelihood function $\mathcal{L}$ is unimodal, and hence $\widehat{\alpha}^{(\ell)}$ converges to the incomplete data MLE provided that $\mathcal{K}\left(\widehat{\gamma}^{(\ell)}\right) \in \mathcal{A}$ for all $\ell$.

\section{Network Inference Algorithm}

In order to carry out inference on measured data, we express the calculation of $\widehat{\gamma}$ in Theorem 5 as an algorithm. We start by constructing $b_{k}, c_{k}$ and $y_{k}^{*}$ recursively. Clearly the $b_{k}$ satisfy

$$
b_{k}= \begin{cases}\bar{\alpha}_{k}, & k \in R \\ \bar{\alpha}_{k}+\alpha_{k} \prod_{j \in d(k)} b_{j}, & k \in U \backslash R\end{cases}
$$

The $c_{k}$ satisfy a similar recursion:

$$
c_{k}= \begin{cases}1, & k \in R, x_{k}^{*}=\mathrm{u} \\ \bar{\alpha}_{k}, & k \in R, x_{k}^{*} \neq \mathrm{u} \\ \bar{\alpha}_{k}+\alpha_{k} \prod_{j \in d(k)} c_{j}, & k \in U \backslash R\end{cases}
$$

The $y_{k}^{*}$ satisfy the recursion

$$
y_{k}^{*}= \begin{cases}x_{k}^{*}, & k \in R \\ \vee_{j \in d(k)}^{*} y_{j}^{*}, & k \in V \backslash R\end{cases}
$$

We formally specify an algorithm for the calculation of the $\widehat{\gamma}_{k, \alpha}$ in Figure 1 . The main procedure comprises two phases. In the first phase, set_ybc, calculates the $y_{k}^{*}, b_{k}$ and $c_{k}$ passing up the tree from the leaves. The second phase, set_g, then calculates the $\widehat{\gamma}_{k, \alpha}$ traversing the tree from the root $\rho$ downwards. $h_{k}$ plays the role of $d(k, h)$ while $e$ plays the role of $\alpha_{i} \prod_{j \in d(i) \backslash\{d(i, k)\}} c_{j}$. On a given path down the tree, flag $=1$ until a node $k$ with $y_{k}^{*}=0$ is first encountered. flag $=0$ on all calls to set_g below $k$. The identity of the node $h\left(i, x^{*}\right)$ is then maintained in calls at nodes $i$ below the child $j$ of $k$ (lines 10-13).

We note there is some redundancy in the algorithms, which can be avoided in implementations. $b_{k}$ and $c_{k}$ need not be calculated at nodes $k$ for which $y_{k}^{*}=1$, since these values are not used. The $c_{k}$ depend only on the missing data indicator $t\left(x^{*}\right)$, and so need be calculated once for each set incomplete outcomes $\left\{x^{*} \in \Omega^{*}: t\left(x^{*}\right)=t\right\}$ having the same missing data indicator $t$. The $b_{k}$ do not depend on $x^{*}$, and so may be calculated once in advance; in particular $b_{k}=c_{k}$ when $x^{*}$ has no missing data, i.e., when $x_{k}^{*} \neq \mathrm{u} \forall k \in R$. Lastly, the $y_{k}^{*}$ need only be calculated once for each probe with distinct $x^{*}$, and once at the start of the sequence of iterations.

\section{Identifiability and Missing Data}

We address the question of identifiability, i.e., whether there exists a unique set of model parameters giving rise to a given distribution of observable data. The multicast inference method exploits correlations between end-to-end measurements on intersection paths. Conversely, we expect that if the sets of receivers on which data from a given probe is observable are insufficiently rich, it will not be possible to infer the loss rates on all links. We give below a simple example that demonstrates this. In this section we shall derive conditionsbetween the topology and the subsets at which data is observable-that must be satisfied in order that the model parameters can be identified. 
1. $\operatorname{procedure} \operatorname{main}\left(\mathcal{T}, \alpha, x^{*}\right)$

2. $\quad \operatorname{set}_{-} \mathrm{ybc}\left(\mathcal{T}, \alpha, x^{*}, \rho\right)$;

3. $\quad \operatorname{set} \_g(\mathcal{T}, \alpha, 1, \rho, \rho, 1)$;

4. $\operatorname{return}\left(\left\{g_{k}: k \in V\right\}\right)$;

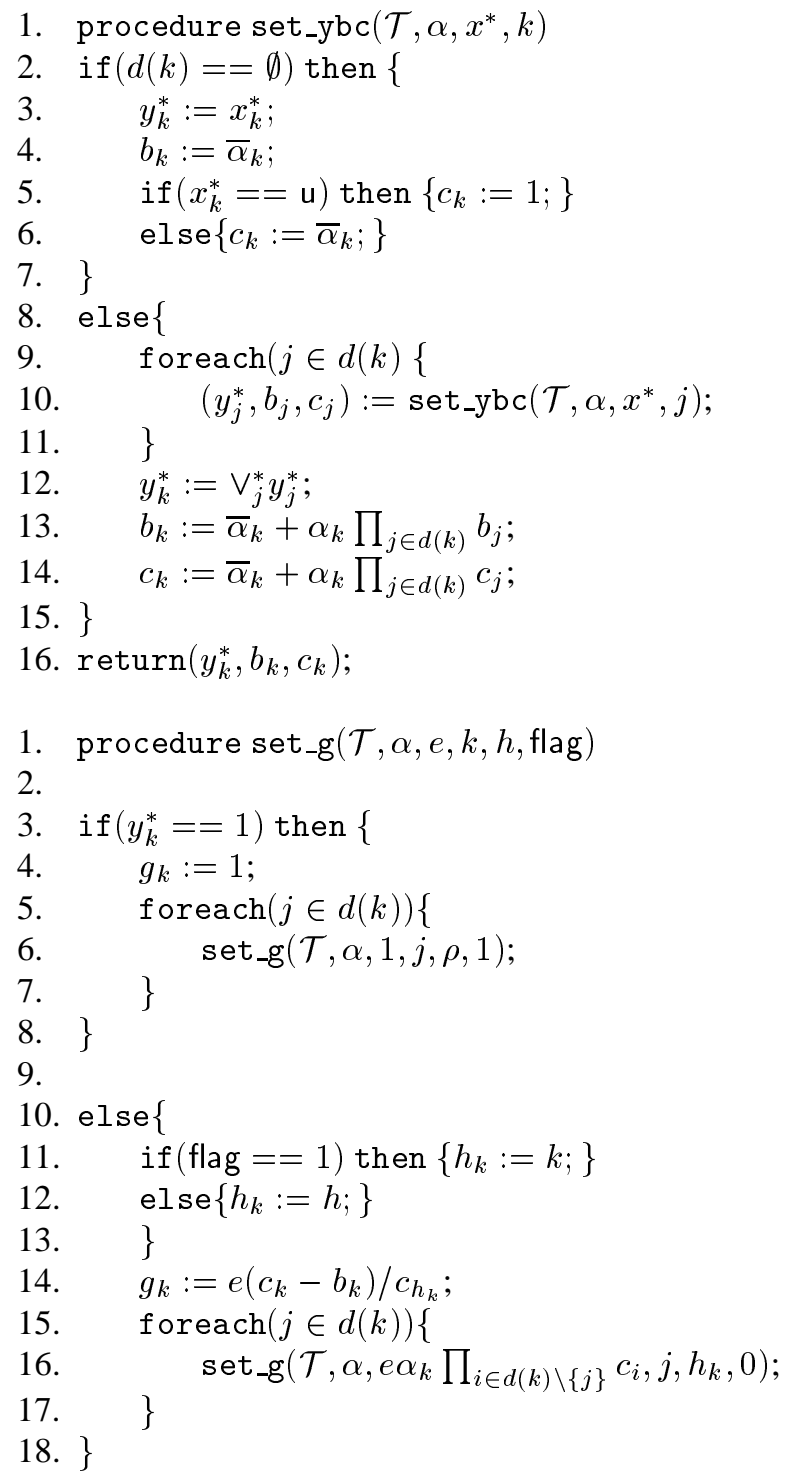

Figure 1: Algorithms for determining $\widehat{\gamma}_{k, \alpha}\left(x^{*}\right)$, as returned from the procedure main. 
Consider a parameterized family of distribution $\left\{\mathrm{P}_{\phi}: \phi \in \Phi\right\}$ with vector parameter $\phi$, and let $F$ be some function on $\Phi$. We say that $\mathrm{P}_{\phi}$ identifies $F(\phi)$ when $\mathrm{P}_{\phi}=\mathrm{P}_{\phi^{\prime}}$ implies $F(\phi)=F\left(\phi^{\prime}\right)$. Here, $F$ will be the identity, or some other projection of components of $\phi$. In an MAR model, $\mathrm{P}_{\alpha, \theta}$ identifies $(\alpha, \theta)$ iff

$$
q_{\alpha, \theta}\left(x^{*}\right)=q_{\alpha^{\prime}, \theta^{\prime}}\left(x^{*}\right), \forall x^{*} \in \Omega^{*} \Rightarrow(\alpha, \theta)=\left(\alpha^{\prime}, \theta^{\prime}\right) .
$$

A simple example of MCAR data that is not identifiable is a two leaf tree in which, for each probe independently, data is missing from exactly one leaf. Then the only non-trivial equations (17) become

$$
\begin{array}{ll}
q_{\alpha, \theta}(1 \mathrm{u})=\alpha_{1} \alpha_{2} \theta(1 \mathrm{u}), & q_{\alpha, \theta}(0 \mathrm{u})=\left(1-\alpha_{1} \alpha_{2}\right) \theta(0 \mathrm{u}) \\
q_{\alpha, \theta}(\mathrm{u} 1)=\alpha_{1} \alpha_{3} \theta(\mathrm{u} 1), & q_{\alpha, \theta}(\mathrm{u} 0)=\left(1-\alpha_{1} \alpha_{3}\right) \theta(\mathrm{u} 0)
\end{array}
$$

The RHS of these equations are invariant w.r.t. the transformations $\alpha_{1} \mapsto k \alpha_{1}, \alpha_{2} \mapsto \alpha_{2} / k, \alpha_{3} \mapsto \alpha_{3} / k$.

With each $S \subset R$ we associate the minimal logical multicast tree $\mathcal{T}_{S}=\left(V_{S}, L_{S}\right)$ that spans the root $\rho$ and $S$. This is obtained by first finding the minimum spanning tree of $\rho$ and $S$ in $\mathcal{T}$. The branch points in the spanning tree, together with $\rho$ and $S$, form the node set $V_{S}$. To define $L_{S}$, the parent $f_{S}(k)$ in $\mathcal{T}_{S}$, of each node in $U_{S}:=V_{S} \backslash\{\rho\}$, is the $\succ$-minimal $j$ in $V_{S}$ such that $j \succ k$ in $\mathcal{T}$. A path in $\mathcal{T}$ that connects two nodes in $V_{S}$ is called an $S$-segment. $K_{S}(i)=\left\{j \in V: i \preceq j \prec f_{S}(i)\right\}$ is the $S$-segment terminating at $i \in U_{S}$. Given $i \in V, \kappa_{S}(i)$ denote the node in $V_{S}$ that terminates the $S$-segment containing $i$, i.e,. that for which $i \in K_{S}\left(\kappa_{S}(i)\right)$. Likewise, $\alpha_{S}(i)=\prod_{j \in K_{S}(i)} \alpha_{j}$ denotes the composite transmission probability along the segment $K_{S}(i) . \alpha_{S}=\left\{\alpha_{S}(i): i \in U_{S}\right\}$ will denote the collection of such probabilities.

Let $D_{S}$ be the $\# U_{S} \times \# U$ incidence matrix of the nodes of $U$ in the segments of $\mathcal{T}_{S}$, i.e., $D_{S, j k}=1$ if $k \in K_{S}(j)$ and 0 otherwise. Setting $\beta_{S}(k)=\log \alpha_{S}(k)$ and $x_{k}=\log \alpha_{k}$ we have that

$$
\beta_{S}=D_{S} x
$$

for any $S \subset R$. Before stating and proving results on identifiability, we note that there exists at least one solution, $\log \alpha$, to (46). Let $\mathrm{P}_{\alpha, \theta, S}$ denote the distribution of the reports from nodes in $S$. We give two conditions for identifiability of $\alpha$.

Theorem 7 Let $\mathcal{T}$ be a canonical loss tree and $\left\{S_{i}\right\}_{i=1}^{m}$ a collection of subsets of $R$.

(i) $U=\cup_{i=1}^{n} U_{S_{i}}$ if and only if the equations $\left\{\beta_{S_{i}}=D_{S_{i}} x\right\}_{i=1}^{m}$ have a unique solution $x$.

(ii) Assume $\mathrm{P}_{\alpha, \theta, S_{i}}$ identifies $\alpha_{S_{i}}$ for each $i$. Then $\left\{\mathrm{P}_{\alpha, \theta, S_{i}}\right\}_{i=1}^{m}$ identifies $\alpha$ iff either (and hence both) of the conditions of part (i) are satisfied.

Remarks. Uniqueness of the solution to (46) is determined by the structure of the $D_{S}$, which depend only on the topology and the choice of the $S$, not on $\beta_{S}$. Consequently, when uniqueness holds, it does so for any additive metric. Thus one can devise a test for identifiability based on path length in terms of number of links. Furthermore, if $\alpha$ is not identifiable, the procedure can be modified to determine which links can be solved.

We say that complete data is available from a subset $S$ if $\theta\left(x^{*}\right)>0$ for all $x^{*}$ such that $R\left(\rho, x^{*}\right)=S$, i.e,. for which reports are received from all receivers in $S$ and no others. Let $\mathcal{S}_{\theta}$ denote the set of subsets 
$S$ of $R$ for which complete data is available, and $\Theta_{c}$ denote the set of missingness parameters $\theta$ for which $U=\cup_{S \in \mathcal{S}} U_{S}$.

Theorem 8 Restrict the parameter space to $\mathcal{A} \times \Theta_{c}$ and assume data is MCAR. Then $\mathrm{P}_{\alpha, \theta}$ identifies $(\alpha, \theta)$.

Although we do not have a corresponding result for general MAR models, Theorem 8 is sufficient to enable further analysis of simple models in the following sections.

\section{Asymptotics for Large Numbers of Probes}

Let $\check{\alpha}=\arg \max _{\alpha} \mathcal{L}(\alpha)$ denote the incomplete data MLE arising from (20). In this section we examine the asymptotic properties of $\check{\alpha}$ as the number of probes $n$ grows, without specific reference to the EM algorithm.

Theorem 9 Assume data is MCAR. The incomplete data MLE $\check{\alpha}$ is consistent, i.e., $\lim _{n \rightarrow \infty} \check{\alpha}=\alpha$ almost surely.

We now describe the asymptotic variance of $\check{\alpha}$ for large numbers of probes $n$ in the regime of small loss probabilities $\bar{\alpha}$. We calculate the expected Fisher information matrix for the incomplete data problem, i.e., the matrix $\mathcal{I}(\alpha, \theta)=\left[\mathcal{I}^{i j}(\alpha, \theta)\right]_{i j \in U}$, where $\mathcal{I}^{i j}(\alpha)=-\mathrm{E} \frac{\partial^{2} \mathcal{L}(\alpha)}{\partial \alpha_{i} \partial \alpha_{j}}$. Under conditions that we establish below, the inverse of $\mathcal{I}(\alpha)$, suitably rescaled, is the asymptotic variance of $\check{\alpha}$.

Our approach is to decompose the Fisher information matrix as a sum over subtrees for which complete data is present at the leaves. In the original incomplete data problem for the logical multicast topology $\mathcal{T}$, the counts $\boldsymbol{n}_{S}=\left\{m\left(x^{*}\right) \mid R\left(\rho, x^{*}\right)=S\right\}$, for each $S \subset R$, can be considered as a set of counts of complete outcomes on $\mathcal{T}_{S}$ stemming from those probes for which reports were received only from nodes in $S$. Thus the incomplete data log-likelihood function can then be decomposed as follows:

$$
\mathcal{L}(\alpha)=\sum_{S \subset R: S \neq \emptyset} \mathcal{L}_{c}\left(\mathcal{T}_{S}, \boldsymbol{n}_{S}, \alpha_{S}\right), \quad \text { where } \quad \mathcal{L}_{c}\left(\mathcal{T}_{S}, \boldsymbol{n}_{S}, \alpha_{S}\right)=\sum_{x^{*}: R\left(\rho, x^{*}\right)=S} m\left(x^{*}\right) \log p_{\alpha_{S}}\left(x_{S}^{*}\right)
$$

and $x_{S}^{*}=\left\{x_{k}^{*}: k \in S\right\}$ is the data in $x^{*}$ that is observable at $S$. The corresponding decomposition of the expected Fisher information matrix is

$$
\mathcal{I}^{i j}(\alpha)=\sum_{S \subset R: S \neq \emptyset} \sum_{k \ell \in U_{S}} \mathcal{I}_{S}^{k \ell}\left(\alpha_{S}\right) \frac{\partial \alpha_{S}(k)}{\partial \alpha_{i}} \frac{\partial \alpha_{S}(\ell)}{\partial \alpha_{j}}
$$

where $\mathcal{I}_{S}^{j k}\left(\alpha_{S}\right)=-\mathrm{E} \frac{\partial^{2} \mathcal{L}_{c}\left(\mathcal{T}_{S}, \boldsymbol{n}_{S}, \alpha_{S}\right)}{\partial \alpha_{S}(j) \partial \alpha_{S}(k)}$. Let $N_{S}=n \mathrm{P}\left[R\left(T^{(i)}\left(X^{(i)}\right)\right)=S\right]$ be the mean number of probes with data observable exactly at $S$, and $W_{S}(i)=\sum_{j \in K_{S}\left(\kappa_{S}(i)\right)} \bar{\alpha}_{j}$ the sum of link loss rates on the $S$-segment containing $i$.

Theorem 10 (i) When $\theta \in \Theta_{c}$ and hence when $\check{\alpha}$ is consistent, $\sqrt{n}(\check{\alpha}-\alpha)$ converges in distribution to a mean zero multivariate Gaussian random variable with covariance matrix $n^{-1} \mathcal{I}(\alpha)$.

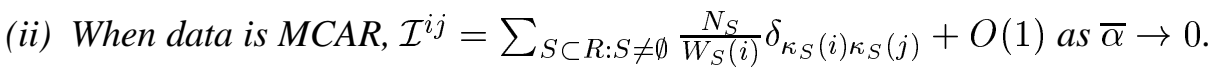


Example: uniform report transmission. Let reports be transmitted independently with uniform probability $p \in(0,1]$. Then $N_{S}=n p^{\# S} \bar{p}^{\# R-\# S}$. For each $S \subset R$, and node $\ell \in U_{S}$, let $C_{S}(\ell)$ denote the matrix on $U$ with entries $C_{S}^{i j}(\ell)=1 / W_{S}(i)$ if $i, j \in K_{S}(\ell)$ and 0 otherwise. For $s \in\{1, \ldots, \# R\}$ let $C_{s}=\sum_{S: \# S=s} \sum_{\ell \in U_{S}} C_{S}(\ell)$. Then

$$
\mathcal{I}=n C \cdot(1+O(\bar{\alpha})), \quad \text { where } \quad C=\sum_{s=1}^{\# R} p^{s}(1-p)^{\# R-s} C_{s}
$$

Let $P_{K}$ denote the orthogonal projection onto the nullspace of a symmetric matrix $K$, and recursively define matrices $K_{1}, \ldots, K_{\# R}$ by $K_{1}=C_{1}$, and

$$
K_{s}=P_{K_{1}+\ldots K_{s-1}} C_{s} P_{K_{1}+\ldots K_{s}}, \quad K_{1}=C_{1} .
$$

Let $r_{0}$ denote the minimal $s$ for which $P_{K_{1}+\ldots+K_{s}}=1$. Since $C_{\# R}=1$, such a $r_{0} \leq \# R$ exists.

Proposition $1 p^{r_{0}} C^{-1}$ converges to the pseudo-inverse of $K_{r_{0}}$ as $p \rightarrow 0$.

Let $I_{2}$ denote the Fisher information arising from measurements on binary subsets, i.e., $I_{2}$ is the sum obtained by restricting (48) to binary subsets $S$.

Proposition 2 (i) $\mathcal{I} \geq \widetilde{\mathcal{I}}_{2}>0$, and hence $0<\mathcal{I}^{-1} \leq \mathcal{I}_{2}^{-1}$, in the order of positive linear operators.

(ii) Proposition 1 holds with $r_{0}=2$.

Thus we have established:

Proposition 3 Assume independent report loss with uniform probability $p$. Then $\sqrt{n}(\check{\alpha}-\alpha)$ converges to a multivariate Gaussian random variable with mean zero and covariance $G(\alpha, p)$, where $\lim _{p \rightarrow 0} p^{2} G(\alpha, p)=$ $K I_{2}+O\left(\|\bar{\alpha}\|^{2}\right)$ as $\bar{\alpha} \rightarrow 0$, where $K I_{2}$ is the pseudo-inverse of $K_{2}$.

Remark: Proposition 3 suggests that we approximate the variance of $\check{\alpha}_{k}$ by $\left(K I_{2}\right)_{k k} /\left(n p^{2}\right)$ when $p$ and $\bar{\alpha}$ are small, and $n$ is large.

Example: uniform report transmission from binary trees Consider the family of binary trees with $2^{r}$ leaves, $r=1,2, \ldots$, with small uniform link loss probabilities $\bar{\alpha}$ and uniform small report transmission rate $p$. Let $v(r)=\left(v_{1}(r), \ldots, v_{r+1}(r)\right)$ denote the set of unique diagonal elements of $K I_{2} / \bar{\alpha}$, the $j^{\text {th }}$ element determining the asymptotic variance on links $j$ nodes away from the root. Using Mathematica [16] to perform the algebra, we found the first six $v(r)$ to be:

$$
\begin{array}{r}
v(1)=\left\{\frac{1}{3}, \frac{1}{3}\right\}, \quad v(2)=\left\{\frac{1}{8}, \frac{21}{40}, \frac{2}{5}\right\}, \quad v(3)=\left\{\frac{3}{80}, \frac{49}{240}, \frac{25}{42}, \frac{3}{7}\right\}, \quad v(4)=\left\{\frac{1}{96}, \frac{43}{672}, \frac{27}{112}, \frac{91}{144}, \frac{4}{9}\right\}, \\
v(5)=\left\{\frac{5}{1792}, \frac{33}{1792}, \frac{5}{64}, \frac{21}{80}, \frac{36}{55}, \frac{5}{11}\right\}, \quad v(6)=\left\{\frac{3}{4096}, \frac{187}{36864}, \frac{133}{5760}, \frac{153}{1760}, \frac{73}{264}, \frac{209}{312}, \frac{6}{13}\right\}
\end{array}
$$

In all cases the estimator variance rises in a given tree on moving away from the root, except falling slightly at a leaf link as compared with its parent. At a given distance from the root, the link variance decreases as the tree depth increases. Both this trends can be understood by the intuition that variance should decrease 


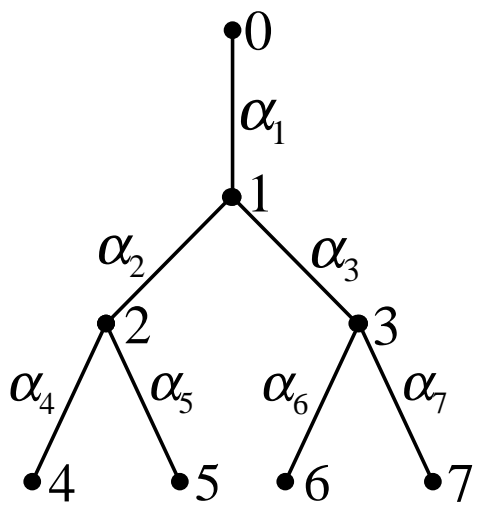

Figure 2: 4-RECEIVER BINARY TREE: used in model based simulation of Section 8.1.

when data is available from larger subtrees below a given link of interest. Considering the root and leaf links only, the values in (51) are consistent with the forms

$$
v_{1}(r)=\frac{r}{r+2} \frac{1}{2^{2(r-1)}}, \quad v_{r+1}(r)=\frac{r}{2 r+1}
$$

\section{Convergence Rates for the EM iterates}

We now consider convergence of the EM iterates themselves. Let $\mathcal{M}$ denote the map on $\mathbb{R}_{+}^{U}$ that implements the iteration, i.e., such that $\widehat{\alpha}^{(\ell+1)}=\mathcal{M}\left(\widehat{\alpha}^{(\ell)}\right)$. A Taylor expansion of the iterative map gives

$$
\widehat{\alpha}^{(\ell+1)}-\alpha \approx \nabla \mathcal{M} \cdot\left(\widehat{\alpha}^{(\ell)}-\alpha\right)
$$

where $\nabla M_{i j}=\frac{\partial \mathcal{M}_{i}}{\partial \alpha_{j}}$ is the gradient of $\mathcal{M}$. A standard result $[17, \S 3.9 .3]$ expresses $\nabla \mathcal{M}=\left(1-\mathcal{I}_{c}^{-1} \mathcal{I}\right)$, with $\mathcal{I}_{c}$ the complete data information matrix and $\mathcal{I}$ the incomplete data information matrix from (48). The convergence ratio of the iteration is taken as the maximum eigenvalue $\lambda$ for $\nabla \mathcal{M}$.

Our analysis of the convergence ratio is confined to the regime treated in Section 6, namely that of independent report transmission with small probability $p$, and small link loss probabilities $\bar{\alpha}$. In this regime, we have seen that $\left(\mathcal{I}_{c}^{-1}\right)_{i j}=n^{-1}\left(\bar{\alpha}_{i} \delta_{i j}+O\left(\|\bar{\alpha}\|^{2}\right)\right.$ and so from (49) $\nabla \mathcal{M}(\alpha)_{i j}=\delta_{i j}-\bar{\alpha}_{i} C_{i j}+O\left(\|\bar{\alpha}\|^{2}\right)$. Let $\mathcal{E}(X)$ denote the set of eigenvalues of a matrix $X$.

Proposition 4 Assume independent report loss with probability $p \in(0,1)$ and small uniform probe loss rate $\bar{\alpha}$. The convergence rate $\lambda$ for the EM algorithm obeys $\lambda=1-p^{2} \kappa+O\left(p^{2}(p+\bar{\alpha})\right)$. where $\kappa$ is the minimum non-zero eigenvalue of $\bar{\alpha} K_{2}$.

\section{Experiments and Simulations}

In order to evaluate the performance of the missing data inference algorithm, we conducted two types of simulation. First, we used model-based simulation in which the model for missing data indicators conformed 
with the MCAR property. Second, we used a network-based implementation of the RTCP-based reporting mechanism outlined in Section 2.5. In this case the missing data indicators are not known to conform to the MAR model. This enabled us to test robustness of the algorithm with respect to violations of the MAR hypothesis that might occur in a real network application.

\subsection{Model-based simulation}

We conducted model based simulations on a balanced binary tree with 4 receivers, illustrated in Figure 2. Probe losses were independent with a uniform loss rate per link. Receiver reports were generated at each receiver for each probe and were transmitted independently with uniform probability $p$. We conducted 100 separate simulation runs, each of 100,000 probes. Initialization of $\widehat{\alpha}^{(0)}$ used (29). The termination criterion

for the EM algorithm was that successive iterates $\widehat{\alpha}_{k}^{(\ell)}$ should have an absolute difference of less than $10^{-4}$ on each link $k$.

Figure 3(left) shows the mean and error bars for 95\% confidence of link loss rate estimates obtained using the missing data algorithm. We also display the corresponding quantities for the complete data estimator applied to only those probes for which complete reports were available. In both cases the mean estimate is close to the model loss rate, i.e. $\bar{\alpha}_{k}=0.01$. But note the rapid widening of error bars for the full data algorithm, compared with the missing data algorithm, as the report transmission probability decreases. From Prop. 3 we expect the standard error of the link loss rate estimates to diverge as $p^{-1}$ for the missing data algorithm, regardless of the topology. However, in a 4-leaf tree the number of probes with complete data is proportional to $p^{4}$. Hence we expect the standard error to diverge as $p^{-2}$, with faster divergence for trees with more receivers. In this example, for $p$ less than 0.4 , the error bars encompass loss rate 0: the inferred loss from complete becomes statistically indistinguishable from 0.

Figure 3(right) breaks down the standard error of the link loss rate estimates according to the location of the link in the topology links 1,2 and 4 being representative of links respectively 0,1 and 2 links removed from the root. The experimental standard errors show close agreement with the theoretical values obtained by inverting the information matrix $\mathcal{I}$ in (49). We also show the small $p$ approximation obtained using Proposition 3 and the values $v(2)$ from (51). The approximation remains reasonable even for quite large $p$.

\subsection{RTCP-based experiments}

The RTCP-based simulations used data gathered from a network-based implementation of loss reporting. Loss reports are embedded in RTCP feedback packets; any collector listening to these can then perform inference. The basic RTCP reporting mechanism includes only the average loss rate based on sequence numbers of received packets. An extension of the report format allows the inclusion of a binary vector indicating receipt or otherwise of a set of packets.

According to the RTP standard [20], the total report volume over all receivers should not exceed 5\% of the source rate. RTCP clients estimate their share of this based upon the reports they hear from the other receivers, and limit report frequency and size accordingly. Consequently, for a sufficiently large number of receivers, it will not be possible to report on all probes. Missingness arises then by two mechanisms: the omission of certain probes from reporting, and the loss of report packets during transmission to the collector. 

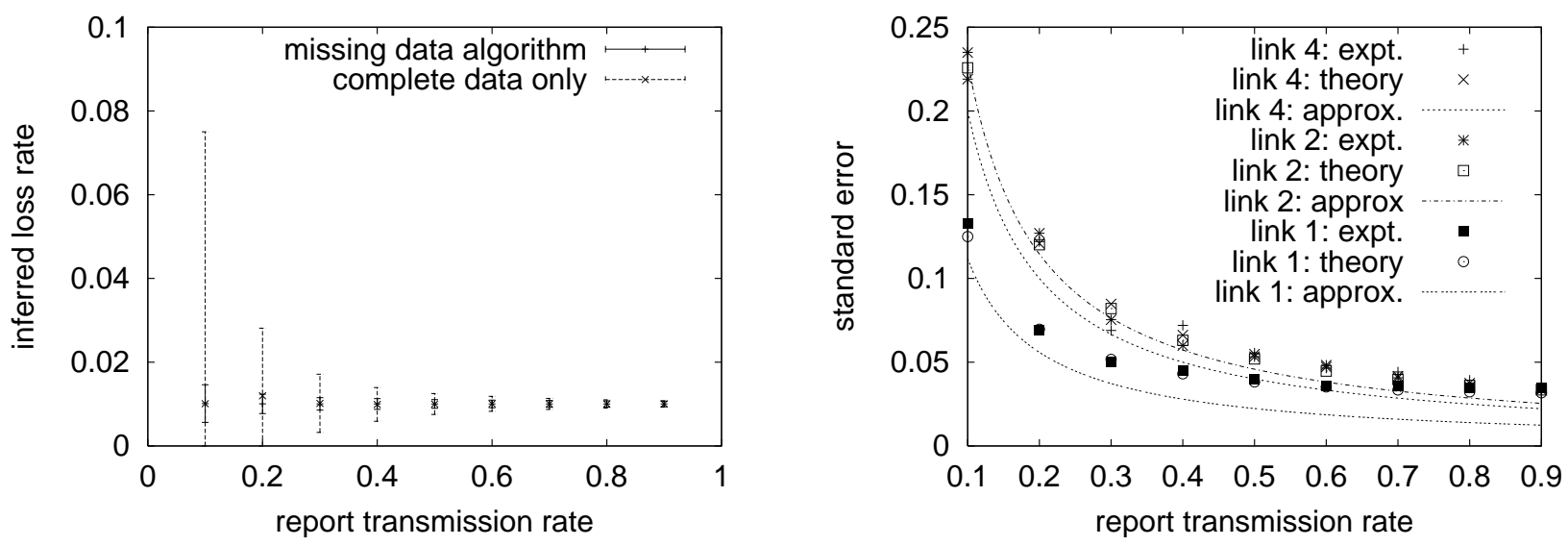

Figure 3: VARIANCE of Missing DATA Estimator in 4-RECEIVER UNIFORM PROBE AND PACKET LOSS MODEL: Over 100 simulation runs each of 100,000 probes, uniform link loss $\bar{\alpha}=0.01$, probe transmission rate $p$ from 0.1 to 0.9 . LEFT: mean estimate with error bars for $95 \%$ confidence. Comparison with estimator using only probes with complete data. RIGHT: standard error depending on link location: experiment, theory and approximation.

The implementation of extended RTCP-based reporting used in this study has a simulation mode that enables it to report on packet losses generated on a model topology according to a Bernoulli loss model, rather than due to packet loss in a real network. The probe source was chosen to have the characteristics of a GSM audio stream that could act as a probe source in real networks, sending packets at a rate of 50 per second. Since probe losses follow the assumed statistical model, only the missing data indicators can potentially exhibit departures from our model assumptions. Report thinning and transmission then takes place in the manner described above.

We collected traces from a 32 receiver balanced binary tree for which the link loss rates were chosen independently with a uniform distribution between $1 \%$ and $10 \%$. The trace comprised reports on 11,956 probe packets, encompassing about 4 minutes at 50 packets per second. The mean number of reports received for a given probe was 18.8 , so that the proportion of missing reports was $1-18.8 / 32=0.413$. The maximum number of reports per probe was 29 , i.e. no probe had complete data. Figure 4(left) shows a scatter plot of the 63 pairs of (actual,inferred) loss rates. The agreement is quiet close, with tight clustering around the line of slope 1 through the origin. The median relative error over all links was only $4.5 \%$.

Figure 4(right) displays the median, $5^{\text {th }}$ and $95^{\text {th }}$ percentile of the relative error over all links as a function of the size of a subset of probes used for inference. Note that even with 2000 probes the relative error is typically less than $50 \%$. Hence we can expect to identify the lossiest links with measurements over a duration less than 1 minute. The median error is only about $13 \%$ for this number of probes. 

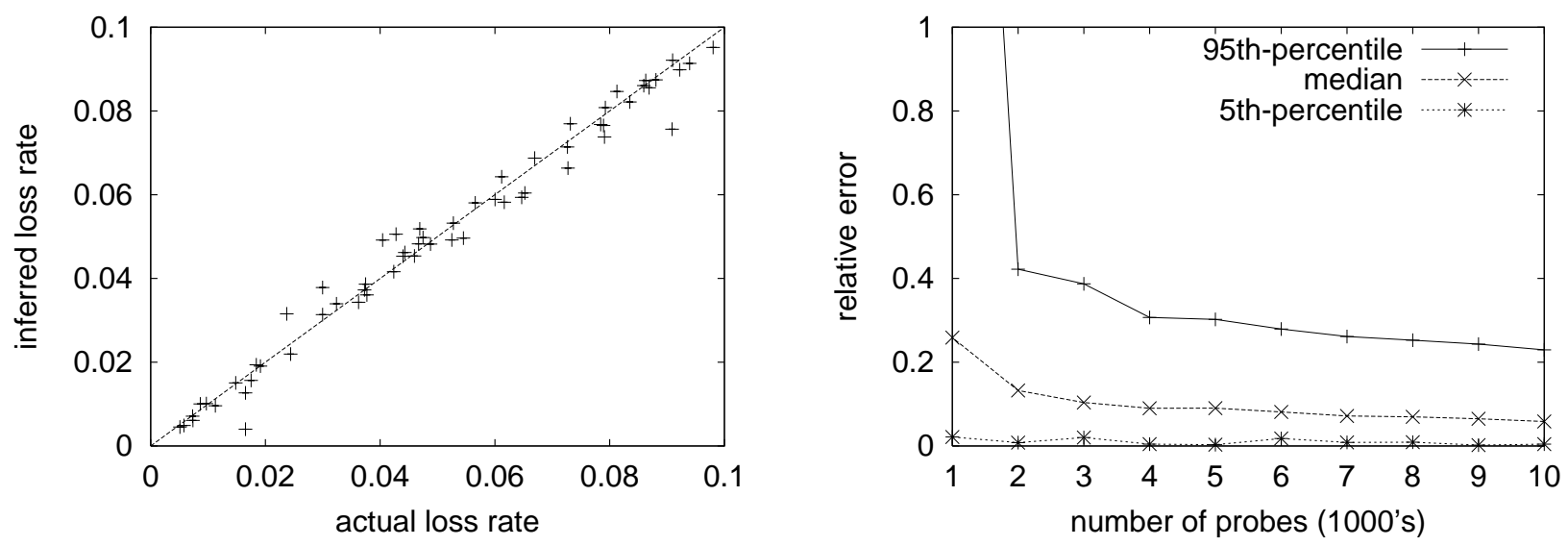

Figure 4: INFERENCE FORM RTCP TRACE DATA ON 32 RECEIVER BINARY TREE. LEFT: Scatter plot of inferred vs. actual loss rates for a full trace of 11,956 probes. RigHT: Median, $5^{\text {th }}$ and $95^{\text {th }}$ percentiles of relative error over all links as function of number of probes.

\section{Conclusions}

In this paper we have extended the multicast based method for inferring network internal loss from end-toend measurements that was first proposed in [2]. The original method assumed the presence of complete data specifying the set of end-points reached by each multicast probe. However, the proposed use of the RTCP transport protocol to transmit measurements inevitably leads to missing data, either through the need to thin data, or due to loss of reports in transmission. This motivated extending the former approach to work with missing data. An ad hoc approach of working with subsets of complete data would have several drawbacks: inference on all links may not be possible; inference would be inconsistent under the types of correlation between probe data and missingness that could reasonably occur in this context (see Section 2.5); and the estimators are not generally efficient. These considerations motivated the use of a more generally applicable scheme that accommodates the missing data directly, under more general conditions on the missing data mechanism.

This paper extended the Maximum-Likelihood approach of [2] to encompass missing data. We applied the EM algorithm to generate an iterative approximation to the corresponding MLE. We analyzed convergence rates for the EM algorithm itself, and for the MLE as the number of probes grows, and showed how to calculate these rates explicitly for a particular class of models. We tested an implementation of the algorithm in model-based simulations with known missingness statistics, and also in traces gathered from an implementation of the RTCP-based report transfer method. These results showed (i) the reduction in estimator variance, as compared with the ad hoc approach, where applicable; and (ii) accuracy of inferred loss rates compared with model or directly measured rates in the simulation; (iii) robustness of the approach under potential departures from the model assumptions on the missingness statistics in the RTCP-based application. In the RTCP-based experiments the median estimation error on a 32 receiver tree was only about $13 \%$ for 2,000 probes, and was typically less than about $50 \%$. Thus inference sufficiently accurate to identify the 
lossiest links could be performed on measurements collected over about a minute.

Future work is planned in two directions. First, we want to apply the same general methodology to the estimation of other internal characteristics, such as delay, utilization and topology itself, by adapting the framework of the present paper to work on estimation of these quantities with complete data, as performed in $[3,9,15]$ A second direction is to develop more specific models of the missing data mechanism that could be used in a parametric approach to estimation with missing data. Lastly, we intend to publish elsewhere details of the RTCP reporting mechanism that motivated this study.

Acknowledgment We thank Francesco Lo Presti for some useful suggestions.

\section{Proofs of Theorems}

Proof of Theorem 2: We first render $\mathcal{L}_{c}(\alpha)$ into the canonical form of a standard exponential family.

- Denote by 0 and 1 the elements $x$ of $\Omega$ with $x_{k}$ all 0 or all 1 respectively.

- For $x \in \Omega$, denote by $W^{\prime}(x)$ those nodes $k \in U$ for which $x_{j}=0$ for all $j \in R(k)$. Let $W(x)$ be the $\succeq$-maximal elements of $W^{\prime}(x)$. Note that $W(\mathbf{1})=\emptyset$.

- For each $k \in U$ and $i \in\{0,1\}$ define $q_{k}(i)=\mathrm{P}_{\alpha}\left[X_{j}=i, \forall j \in R(k) \mid X_{f(k)}=1\right]$.

- Define new parameters $\left\{\delta_{k}: k \in U\right\}$ by $\delta_{k}=\log \left(q_{k}(0) / q_{k}(1)\right)$.

- Observe that

$$
\frac{p_{\alpha}(x)}{p_{\alpha}(\mathbf{1})}=\prod_{k \in W(x)} \frac{q_{k}(0)}{q_{k}(1)}=\prod_{k \in W(x)} e^{\delta_{k}}
$$

We interpret the product over $\emptyset$ for $x=1$ as 1 .

- The map taking $\mathcal{A}$ to its image $\Delta$ under the change of parameters $\alpha \mapsto \delta$ is invertible. To see this note that given $\sum_{x \in \Omega} p_{\alpha}(x)=1$, (54) fixes the $p_{\alpha}(x)$ in terms of the $\delta$. These in turn determine the $\gamma_{k}$, and hence the $\alpha_{k}$, by Theorem 2 .

- Writing $n(1)=n-\sum_{x \neq \mathbf{1}} n(x)$, and recalling that $W(\mathbf{1})=\emptyset$, we find

$$
\mathcal{L}_{c}(\alpha)=\sum_{x \in \Omega} n(x) \sum_{k \in W(x)} \delta_{k}+n \log p_{\alpha}(\mathbf{1})=\sum_{k \in U} N_{k} \delta_{k}-n c(\delta)
$$

where $N_{k}=\sum_{x \in \Omega: k \in W(x)} n(x)$, and $c(\delta)$ is the reparameterization of $-\log p_{\alpha}(\mathbf{1})$ in terms of $\delta$ :

$$
c(\delta)=\log \sum_{x \in \Omega} \prod_{k \in W(x)} e^{\delta_{k}}
$$

The expression (55) has the form of a standard exponential family, with the log-likelihood expressed in terms of the natural parameters $\delta=\left\{\delta_{k}: k \in U\right\}$, sufficient statistics $N=\left\{N_{k}: k \in U\right\}$, and cumulant 
$c(\delta)$. Since $c(\delta)$ is finite for all $\delta \in \mathbb{R}^{U}$ the family can be considered full. However, the parameter space of interest is the open subset $\Delta \subset \mathbb{R}^{U}$ that is the image of $\mathcal{A}$ under the reparameterization $\alpha \mapsto \delta$.

Since the mapping $\mathcal{A} \rightarrow \Delta: \alpha \mapsto \delta$ is invertible, the parameters $\delta$ are identifiable by Theorem 2(v), and hence the exponential family is affinely independent. (A simple argument shows that natural parameters in an open set are identifiable iff the exponential family is affinely independent). A well-known result (see e.g. [13, Ex. 6.6.3.]) for standard exponential families then says that the MLE is the solution $\delta^{\prime}$ of

$$
N_{k}=\mathrm{E}_{\delta^{\prime}}\left[N_{k}\right], \quad k \in U
$$

provided this $\delta$ lies in the interior of $\Delta$. But clearly $\sum_{j \succeq k} N_{j}=n\left(1-\widehat{\gamma}_{k}\right)$, and hence finding the solution to (56) is equivalent to finding the solution $\alpha^{\prime}$ to

$$
\gamma_{k}=\mathrm{E}_{\alpha^{\prime}}\left[\widehat{\gamma}_{k}\right], \quad k \in U
$$

Provided $\widehat{\gamma} \in \mathcal{G}$, then by Theorem $1, \widehat{\alpha}$ is the unique such solution, and hence if it lies in $\mathcal{A}$ it is the MLE.

Proof of Theorem 3: $\quad$ Observe that $\mathrm{E}_{\alpha}\left[\mathcal{L}_{c}\left(\alpha^{\prime}\right) \mid \boldsymbol{m}\right]=\sum_{x \in \Omega} \mathrm{E}_{\alpha}[n(x) \mid \boldsymbol{m}] \log p_{\alpha^{\prime}}(x)$. Hence maximizing $Q_{\widehat{\alpha}^{(\ell)}, \alpha^{\prime}}$ over $\alpha^{\prime}$ is equivalent to finding the complete-data MLE, but with $n(x)$ replaced by $\mathrm{E}_{\widehat{\alpha}^{(\ell)}}[n(x) \mid \boldsymbol{m}]$ throughout. In particular, $\widehat{\gamma}_{k}$, being a linear combination of the $n(x)$, gets replaced by $\widehat{\gamma}_{k}^{(\ell)}$. Now if $\widehat{\alpha}^{(\ell)} \in \mathcal{A}$ then it is not hard to see that $\widehat{\gamma}_{\widehat{\alpha}^{(\ell)}}\left(x^{*}\right) \in \mathcal{G}$ for each $x^{*}$, and hence $\widehat{\gamma}^{(\ell)} \in \mathcal{G}$ since $\mathcal{G}$ is convex. The result then follows from Theorems 1 and 2.

Proof of Theorem 4: The condition says that the sequence of EM iterates exists in $\mathcal{A}$. Parts (i) and (ii) follow from Theorem 6 of [22]. This is because: (a) by Theorem 1(iii), $\widehat{\alpha}^{(\ell+1)}$ is stationary for $\alpha \mapsto$ $Q\left(\alpha, \widehat{\alpha}^{(\ell)}\right)$; (b) $\nabla_{\alpha} Q\left(\alpha^{\prime}, \alpha\right)$ is clearly continuous for $\left(\alpha^{\prime}, \alpha\right) \in \mathcal{A} \times \mathcal{A}$; and (c) By Theorem 2, $\mathcal{L}_{c}$ comes from a regular exponential family and hence $\left\|\widehat{\alpha}^{(\ell+1)}-\widehat{\alpha}^{(\ell)}\right\| \rightarrow 0$ as $\ell \rightarrow \infty$; see remark 3(vi) in [22]. Part (iii) then follows from Corollary 1 in [22]

Proof of Theorem 5: For the proof we observe the following Markov property of the probe process $X$ :

(M) Conditioned on $X_{i}=1$, the distributions of the sets of variables $\left\{X_{k}: k \in R(i)\right\}$ are independent for different $i \in d(k)$.

If $y_{k}^{*}=1$, then $\vee_{j \in R(k)} x_{j}=1$, and hence $\widehat{\gamma}_{k, \alpha}\left(x^{*}\right)=1$. Suppose instead that $y_{k}^{*}<1$. Let $Q(k)$ denote the event that $X_{j}=0$ for all $j \in R\left(k, x^{*}\right)$ if $R\left(k, x^{*}\right) \neq \emptyset$, otherwise take $Q(k)$ as the universal set in the underling probability space. Since $y_{h}^{*}=1$ for $h=h\left(k, x^{*}\right)$, then $\left\{X^{*}=x^{*}\right\} \subset\left\{X_{h}=1\right\}$ and hence $\left\{X^{*}=x^{*}\right\}=\left\{X_{h}=1\right\} \cap Q(d(h, k)) \cap\left\{X_{j}^{*}=x_{j}^{*}: j \in R \backslash R(d(h, k))\right\}$. This yields that

$$
\mathrm{P}_{\alpha}\left[\vee_{j \in R(k)} X_{j}=1 \mid X^{*}=x^{*}\right]=\frac{\mathrm{P}_{\alpha}\left[\left\{\vee_{j \in R(k)} X_{j}=1\right\} \cap Q(d(k, h)) \mid X_{h}=1\right]}{\mathrm{P}_{\alpha}\left[Q(d(k, h)) \mid X_{h}=1\right]},
$$

where we have used property (M), and the fact that $\mathrm{P}[A \mid B \cap C \cap D]=\mathrm{P}[A|C| B]$ when $A$ and $C$ are conditionally independent of $D$ given $B$. 
The denominator in (58) is just $c_{d(h, k)}$. To treat the numerator, observe that

$$
\begin{aligned}
\mathrm{P}_{\alpha} & {\left[\left\{\vee_{j \in R(k)} X_{j}=1\right\} \cap Q(i) \mid X_{f(i)}=1\right] } \\
& =\mathrm{P}_{\alpha}\left[\left\{\vee_{j \in R(k)} X_{j}=1\right\} \cap Q(d(k, i)) \cap\left\{X_{i}=1\right\} \cap_{j \in d(i) \backslash d(k, i)} Q(j) \mid X_{f(i)}=1\right] \\
& =\mathrm{P}_{\alpha}\left[\left\{\vee_{j \in R(k)} X_{j}=1\right\} \cap Q(d(k, i))\left|X_{i}=1\right| X_{f(i)}=1\right] \mathrm{P}_{\alpha}\left[X_{i}=1 \mid X_{f(i)}=1\right] \prod_{j \in d(i) \backslash d(k, i)} \mathrm{P}_{\alpha}\left[Q(j)\left|X_{i}\right| X_{f(i)}\right] \\
& =\mathrm{P}_{\alpha}\left[\left\{\vee_{j \in R(k)} X_{j}=1\right\} \cap Q(d(k, i)) \mid X_{i}=1\right] \alpha_{i} \prod_{j \in d(i) \backslash d(k, i)} c_{j} .
\end{aligned}
$$

Here we have used $Q(j)=\cap_{i \in d(j)} Q(i)$, the Markov property (M), and the fact that $\left\{X_{k}=1\right\} \subset$ $\left\{X_{f(k)}=1\right\}$. Applying (59) repeatedly to the numerator of (58), we obtain the form $\mathrm{P}_{\alpha}\left[\left\{\vee_{j \in R(k)} X_{j}=\right.\right.$ $\left.1\} \cap Q(k) \mid X_{f(k)}=1\right] \prod_{k \prec i \preceq d(k, h)} \alpha_{i} \prod_{j \in d(i) \backslash\{d(i, k)\}} c_{j}$.

The first term is in this product is $\mathrm{P}_{\alpha}\left[\left\{\vee_{j \in R(k)} X_{j}=1\right\} \cap Q(k) \mid X_{f(k)}=1\right]=c_{k} \mathrm{P}_{\alpha}\left[\left\{\vee_{j \in R(k)} X_{j}=\right.\right.$ 1\} $\left.|Q(k)| X_{f(k)}=1\right]=c_{k}\left(1-\mathrm{P}_{\alpha}\left[\vee_{j \in R(k)} X_{j}=0 \mid X_{f(k)}=1\right] / c_{k}\right)=c_{k}-b_{k}$, and the stated result (35) follows.

Proof of Lemma 1: We first show that for each $k \in V$ there is some $x^{*} \in \Omega_{0}^{*}$ with $m\left(x^{*}\right)>0$ for which $\widehat{\gamma}_{k, \alpha}\left(x^{*}\right)>0$. By condition (38) there is $x^{*}$ with $m\left(x^{*}\right)>0$, for which either $x_{j}^{*}=1$ or $x_{j}^{*}=\mathrm{u}$ for some $j \in R(k)$. In the former case $\widehat{\gamma}_{k, \alpha}\left(x^{*}\right)=1-$ or $x_{j}^{*}=\mathrm{u}$; in the latter case it is not hard to see in Theorem 5 that $c_{k}>b_{k}$ and hence $\widehat{\gamma}_{k, \alpha}\left(x^{*}\right)>0$. Since $\widehat{\gamma}_{k, \alpha}\left(x^{*}\right) \geq 0$ for all $x^{*} \in \Omega_{0}^{*}$, then $\widehat{\gamma}_{k, \alpha}>0$.

By (40) then for each $k \in V \backslash R$ there exists $x^{*}$ with $m\left(x^{*}\right)>0$ such that $\widehat{\gamma}_{k, \alpha}\left(x^{*}\right)=1$, and children $j, \ell$ of $k$ for which $\widehat{\gamma}_{j, \alpha}\left(x^{*}\right)=1$ while $\widehat{\gamma}_{j, \alpha}\left(x^{*}\right)>0$. Hence $\widehat{\gamma}_{k, \alpha}\left(x^{*}\right)<\sum_{j \in d(k)} \widehat{\gamma}_{j, \alpha}\left(x^{*}\right)$. Since by definition $\widehat{\gamma}_{k, \alpha}\left(x^{*}\right) \leq \sum_{j \in d(k)} \widehat{\gamma}_{j, \alpha}\left(x^{*}\right)$ for all $x^{*}$, we have $\widehat{\gamma}_{k, \alpha}<\sum_{j \in d(k)} \widehat{\gamma}_{j, \alpha}$.

Taking these relations over all relevant $k$, we conclude that $\widehat{\gamma}_{\alpha} \in \mathcal{G}$.

Proof of Theorem 6: $\quad$ Reparameterizing the incomplete data likelihood function $\mathcal{L}$ in terms of $\gamma$, we obtain

$$
\begin{aligned}
\mathcal{L}= & n(11) \log \left(\gamma_{2}+\gamma_{3}-\gamma_{1}\right)+n(10) \log \left(\gamma_{1}-\gamma_{3}\right)+n(01) \log \left(\gamma_{1}-\gamma_{2}\right)+n(00) \log \left(1-\gamma_{1}\right) \\
& +n(1 \mathbf{u}) \log \left(\gamma_{2}\right)+n(\mathbf{u} 1) \log \left(\gamma_{3}\right)+n(0 \mathbf{u}) \log \left(1-\gamma_{2}\right)+n(\mathbf{u} 0) \log \left(1-\gamma_{3}\right)
\end{aligned}
$$

Writing this form as $\mathcal{L}=\sum_{x * \in \Omega_{0}^{*}} n\left(x^{*}\right) \mathcal{L}_{x^{*}}$, it suffices to show that $-\mathcal{L}_{x^{*}}$ is jointly convex in $\gamma_{1}, \gamma_{2}, \gamma_{3}$ for each $x^{*}$. This follows from the fact, established by direct computation, that the principal minors of the Hessian matrices $-\left[\partial^{2} \mathcal{L}_{x^{*}} / \partial \gamma_{i} \partial \gamma_{j}\right]_{i j=1,2,3}$ are non-negative. Convergence then follows from Theorem 4 and the standing assumptions (38) and (40).

Remark: the method used in the proof appears not to extend to more general trees, not even binary ones.

Proof of Theorem 7: $\quad$ (i) We establish that if $U=\cup_{i=1}^{m} U_{S_{i}}$, then there is a unique solution $x$. We do this by contradiction. Suppose that there exists a node $k \in V$ such that there does not exist a unique 
value for $x_{k}$. Pick any such $\prec$-maximal $k$. By assumption, $k$ terminates some segment $K_{S_{i}}(k)$ and hence $x_{k}=\beta_{S_{i}}(k)-\sum_{k \prec j \prec f_{S_{i}}(k)} x_{j}$. By the maximality assumption, all terms on the RHS are unique, and hence so is $x_{k}$.

We establish now that if there is a unique solution $x$, then $U=\cup_{i=1}^{m} U_{S_{i}}$. This is done by contradiction. First, note if the solution $x$ is unique, it must be $x_{k}=\log \alpha_{k}$. Second, note that we only need to consider a branch point $k \in U \backslash R$. Assume there exists $k \notin \cup_{i=1}^{m} U_{S_{i}} \backslash R$. Then every segment containing $v \in d(k)$ also contains $k$. Using $x_{k}=\log \alpha_{k}$ we can reduce the family of equations $\left\{\beta_{S_{i}}=D_{S_{i}} x\right\}_{i=1}^{m}$ to the following set of equations describing the behavior of $x_{k}$ and $x_{v}, v \in d(k)$,

$$
x_{k}+x_{v}=\beta_{v}^{\prime}, \quad \forall v \in d(k)
$$

where $\beta_{v}^{\prime}$ is the log of the probability of a reception of a packet at $v \in d(k)$ given that it was received at $f(k)$. The $\beta_{v}^{\prime}$ are determined by the link probabilities other than $\alpha_{k}$ and $\alpha_{v}, v \in d(k)$, and the original $\beta_{S_{i}}$. However, these equations do not have a unique solution, resulting in a contradiction.

(ii) The solution $x$ to $\left\{\beta_{S_{i}}=D_{S_{i}} x\right\}_{i=1}^{m}$ is not unique if and only if there is more that one set of link probabilities $\alpha$ giving rise to the same $\left\{\alpha_{S_{i}}\right\}_{i=1}^{m}$ and hence the same $\left\{\mathrm{P}_{\alpha, \theta, S_{i}}\right\}_{i=1}^{m}$.

Proof of Theorem 8: With MCAR the missingness probabilities do not depend on any data, and so we can replace $\theta\left(x^{*}\right)$ with $\theta\left(t\left(x^{*}\right)\right)$. Since $\sum_{x^{*}: t\left(x^{*}\right)=t^{\prime}} p_{\alpha}^{*}\left(x^{*}\right)=1$ for all $t^{\prime} \in\{0,1\}^{R}, q_{\alpha, \theta}\left(x^{*}\right)=$ $q_{\alpha^{\prime}, \theta^{\prime}}\left(x^{*}\right), \forall x^{*} \in \Omega^{*}$ implies $\theta=\theta^{\prime}$.

Consider now any $S \subset R$ for which complete data is available. Then $\theta\left(t\left(x^{*}\right)\right)$ are equal and hence strictly positive for any $x^{*}$ with $R\left(\rho, x^{*}\right)=S$. Hence $q_{\alpha, \theta}\left(x^{*}\right)=q_{\alpha^{\prime}, \theta^{\prime}}\left(x^{*}\right)$ implies $p_{\alpha}\left(x^{*}\right)=p_{\alpha^{\prime}}\left(x^{*}\right)$. By Theorem 1(iv), $\alpha_{S}$ is identifiable. Then $\alpha$ is identifiable by Theorem 7(ii) since $\theta \in \Theta_{c}$.

Proof of Theorem 9: The proof of mirrors that of Theorem 3(ii) in [2] which in turn uses Lemma 7.54 in [19]. Although not mentioned there, the latter result requires identifiability. This follows from the MCAR assumption and Theorem 8 .

Proof of Theorem 10: We first show that $\mathcal{I}_{S}$ is positive definite if complete data is available from $S$. By standard arguments (see e.g. Prop 2.84 in [19]) one writes $\mathcal{I}_{S}^{j k}\left(\alpha_{S}\right)=\operatorname{Cov}_{\alpha, \theta}\left(\frac{\partial \mathcal{L}_{c}\left(\mathcal{T}_{S}, \boldsymbol{n}_{S}, \alpha_{S}\right)}{\partial \alpha_{S}(j)}, \frac{\partial \mathcal{L}_{c}\left(\mathcal{T}_{S}, \boldsymbol{n}_{S}, \alpha_{S}\right)}{\partial \alpha_{S}(k)}\right)$. If $\mathcal{I}_{S}^{j k}\left(\alpha_{S}\right)$ is not positive definite, there exists some nonzero $c \in \mathbb{R}^{U_{S}}$ for which $c \cdot \mathcal{I}_{S}\left(\alpha_{S}\right) \cdot c=\operatorname{Var}_{\alpha, \theta}(c$. $\left.\frac{\partial \mathcal{L}_{c}\left(\mathcal{T}_{S}, \boldsymbol{n}_{S}, \alpha_{S}\right)}{\partial \alpha_{S}}\right)=0$. This happens if $c \cdot \frac{\partial \mathcal{L}_{c}\left(\mathcal{T}_{S}, \boldsymbol{n}_{S}, \alpha_{S}\right)}{\partial \alpha_{S}}=0$, almost surely, or, equivalently, if $c \cdot \frac{\partial \log p_{\alpha_{S}}\left(x_{S}^{*}\right)}{\partial \alpha_{S}}=$ 0 for all $x_{S}^{*}$, since $\theta\left(x^{*}\right)>0$ by assumption. Repeating the argument of Theorem 4(ii) in [2], this requires $c=0$.

Observe that $\frac{\partial \alpha_{S}}{\partial \alpha}=B\left(\alpha_{S}\right) D_{S} B^{-1}(\alpha)$ where $B(\alpha)$ is the diagonal matrix with entries from $\alpha$. Thus $\mathcal{I} \geq \sum_{S \in \mathcal{S}_{\theta}} B^{-1}(\alpha) D_{S}^{T} B\left(\alpha_{S}\right) I_{S}\left(\alpha_{S}\right) B\left(\alpha_{S}\right) D_{S} B^{-1}(\alpha)$, in the order of positive linear operators. Since the kernel of a sum of non-negative definite operators is the intersection of their kernels, the sum is positive definite iff $\cap_{S \in \mathcal{S}_{\theta}} \operatorname{ker}\left(D_{S}\right)=\{0\}$, which happens iff the equations $\left\{\beta_{S}=D_{S} x\right\}_{S \in \mathcal{S}_{\theta}}$ have a unique solution $x$, which is guaranteed by Theorem 7(i) and the assumption that $\theta \in \Theta_{c}$. 
(ii) Let $S \in \mathcal{S}_{\theta}$. Note $\alpha_{S}(k) / \alpha_{i}=D_{S, k i}(1+O(\|\bar{\alpha}\|))$. With data MCAR, the $\theta\left(x^{*}\right)$ are equal for any $x^{*}$ with $R\left(\rho, x^{*}\right)=S$, and hence $\mathrm{E} m\left(x^{*}\right)=N_{S} p_{\alpha}^{*}\left(x^{*}\right)=N_{S} p_{\alpha_{S}}\left(x_{S}^{*}\right)$. From Theorem 5 of [2], $N_{S}^{-1} \mathcal{I}_{S}\left(\alpha_{S}\right)$ has inverse $\nu_{S}$ for which $\nu_{S}^{k \ell}=\bar{\alpha}_{S}(k) \delta_{k \ell}+O\left(\left\|\bar{\alpha}_{S}\right\|^{2}\right)$. Hence $\mathcal{I}_{S}^{k \ell}\left(\alpha_{S}\right)=\frac{N_{S}}{\bar{\alpha}_{S}(k)}\left(\delta_{k \ell}+O\left(\left\|\bar{\alpha}_{S}\right\|\right)\right.$. Finally, observe that $\bar{\alpha}_{S}\left(\kappa_{S}(i)\right)=W_{S}(i)+O\left(\|\bar{\alpha}\|^{2}\right)$. Putting these together with (48) the result follows.

Proof of Proposition 1: Rewrite $C=\sum_{s=1}^{\# R} p^{s} C_{s}^{\prime}$ where $C_{s}^{\prime}=\sum_{1 \leq t \leq s} C_{t} \cdot(-1)^{s-t}\left(\begin{array}{c}n-t \\ s-t\end{array}\right)$. Observe that the definition of $K_{s}$ is invariant under replacement of $C_{s}$ by $C_{s}^{\prime}$ in (50) since $P_{K_{1}+\ldots+K_{s-1}} C_{s^{\prime}} P_{K_{1}+\ldots+K_{s-1}}=$ 0 for $s^{\prime}<s$. Let $W_{s}$ denote the unitary matrix that diagonalizes $K_{s}$ and set $W=\prod_{i=1}^{r_{0}} W_{s}$. (Since the ranges of the $K_{s}$ are disjoint, the various $W_{s}$ commute). Then up to unitary transformation under $W$, we can write $C$ is block diagonal form

$$
C=W\left(\begin{array}{cccc}
p A_{11}(p) & p^{2} A_{12}(p) & \cdots & p^{r_{0}} A_{1 r_{0}}(p) \\
p^{2} A_{21}(p) & p^{2} A_{22}(p) & \cdots & p^{r_{0}} A_{2 r_{0}}(p) \\
\vdots & \vdots & \ddots & \vdots \\
p^{r_{0}} A_{r_{0} 1}(p) & p^{r_{0}} A_{r_{0}}(p) & \cdots & p^{r_{0}} A_{r_{0} r_{0}}(p)
\end{array}\right) W^{T}
$$

where $A_{i j}(p)=A_{j i}^{T}(p)$, and each submatrix $A_{i j}$ converges to some $A_{i j}$ as $p \rightarrow 0$ such that the block matrix with $A_{s s}$ as the $s^{\text {th }}$ diagonal element and zero elsewhere is unitarily equivalent to $K_{s}$ under $W$. By construction, the $A_{s s}$ are invertible, and hence so are the $A_{s s}(p)$ for sufficiently small $p$. The block diagonal representation of $C$ can be inverted inductively as follows. Assume the block submatrix $B_{[s-1][s-1]}$ comprising the first $s-1$ blocks can be inverted and that $B_{[s-1][s-1]}^{-1}$ is $O\left(p^{1-s}\right)$ as $p \rightarrow 0$. This condition is trivially satisfied for $s=2$. Now write $B_{[s][s]}$ as a two-by-two superblock matrix

$$
B_{[s][s]}=\left(\begin{array}{cc}
B_{[s-1][s-1]} & B_{[s-1] s} \\
B_{s[s-1]} & p^{s} A_{s s}(p)
\end{array}\right)
$$

Then $B_{[s][s]}^{-1}=D_{s, 1}(p) \cdot D_{s, 2}^{-1}(p)$ where

$$
\begin{aligned}
D_{s, 1}(p) & =\left(\begin{array}{cc}
B_{[s-1][s-1]}^{-1} & -p^{-s} B_{[s-1][s-1]}^{-1} B_{[s-1] s} A_{s s}^{-1}(p) \\
-p^{-s} A_{s s}^{-1}(p) B_{s[s-1]} B_{[s-1][s-1]}^{-1} & p^{-s} A_{s s}^{-1}(p)
\end{array}\right) \\
D_{s, 2}(p) & =\left(\begin{array}{cc}
1-p^{-s} B_{[s-1] s} A_{s s}^{-1}(p) B_{s[s-1]} B_{[s-1][s-1]}^{-1} & 0 \\
0 & 1-p^{-s} B_{s[s-1]} B_{[s-1][s-1]}^{-1} B_{[s-1] s} A_{s s}^{-1}(p)
\end{array}\right)(64)
\end{aligned}
$$

Now $B_{[s-1], s}=O\left(p^{s}\right)$ as $p \rightarrow 0$, from which it follows that

$$
B_{[s, s]}(p)=\left(\begin{array}{cc}
0 & 0 \\
0 & p^{-s} A_{s s}^{-1}
\end{array}\right)+O\left(p^{1-s}\right)
$$

Consequently, $B_{[s s]}^{-1}=O\left(p^{-s}\right)$ as $p \rightarrow 0$, completing the induction step. The statement of the Proposition then follows from (65) by taking $s=r_{0}$.

Proof of Proposition 2: (i) In the given model, $N_{S}>0$ for all subsets $S$ of $R$, and hence $\mathcal{I} \geq \mathcal{I}_{2}$. Since each node in $U \subset R$ has at least two descendent leaves $U=\cup_{\# S=2} U_{S}$ and hence $\mathcal{I}_{2}>0$ by an argument 
similar to that in Theorem 7(i). Now if $A \geq B>0$ then $0<1-a \leq 1-b<1$ where $a=A /(2\|A\|)$ and $b=B /(2\|A\|)$. Since $\|1-a\|,\|1-b\|<1, a^{-1}=(1-(1-a))^{-1}=\sum_{i=0}^{\infty}(1-a)^{i}<\sum_{i=0}^{\infty}(1-b)^{i}=b^{-1}$, whence the result follows.

(ii) Consider inference performed by using only measurements from binary sets $S$. $\mathcal{I}_{2}=p Q_{1}+p^{2} Q_{2}$ for some $Q_{1}$ and $Q_{2}$ independent of $p$, and hence $\mathcal{I}_{2}^{-1}$ is $O\left(p^{-2}\right)$. By (i), $\mathcal{I}^{-1} \leq \mathcal{I}_{2}^{-1}$, which precludes $r_{0}>2$ in Proposition 1. We conclude $r_{0}=2$ by showing that $K_{1}$ has 0 as an eigenvalue, for then $r_{0}>1$. Assume that the root $\rho$ as a unique child 1 . If not, partition the $\mathcal{T}$ into disjoint subtrees with nodes descended from each child of $\rho$, then apply the following argument to each subtree. Let $v$ denote the element of $R^{U}$ with $v(1)=1, v(j)=-1$ for $j \in d(1)$, and $v(k)=0$ otherwise. Observe that for each $k \in R$, the $C_{\{k\}}(k)_{i j}$ are equal for $i, j \in\{1, d(k, 1)\}$. Since $C_{1}=\sum_{k \in R} C_{\{k\}}$, then $C_{1} \cdot v=0$.

Proof of Proposition 4: $\max \mathcal{E}(\nabla \mathcal{M})=1-\bar{\alpha} \min \mathcal{E}(C)+O(\bar{\alpha}))$. From Propositions 1 and 2 we know that $C$ takes the block diagonal form (61) with $r_{0}=2$. From this is follows that each eigenvalue of $C$ takes the form $p^{i} v_{i}(p)+O\left(p^{i+1}\right)$ for some $i \in\{1,2\}$, where $\lim _{p \rightarrow 0} v_{i}(p) \in \mathcal{E}\left(A_{i i}\right)$. Since $0 \notin \mathcal{E}\left(A_{i i}\right)$, $p^{-2} \min \mathcal{E}(C) \rightarrow \min \mathcal{E}\left(A_{22}\right)$ as $p \rightarrow 0$, and hence the result follows. 


\section{References}

[1] A. Adams, T. Bu, R. Cáceres, N.G. Duffield, T. Friedman, J. Horowitz, F. Lo Presti, S.B. Moon, V. Paxson, D. Towsley, The Use of End-to-End Multicast Measurements for Characterizing Internal Network Behavior, IEEE Communications Magazine, May 2000.

[2] R. Cáceres, N.G. Duffield, J.Horowitz and D. Towsley, "Multicast-Based Inference of Network Internal Loss Characteristics" IEEE Trans. on Information Theory, vol. 45, pp. 2462-2480, 1999.

[3] R. Cáceres, N.G. Duffield, J.Horowitz F. Lo Presti, D. Towsley, "Statistical Inference of Multicast Network Topology", in Proc. IEEE Conf. on Decision and Control, Phoenix, AZ, Dec. 1999.

[4] R. Carter, M. Crovella, "Measuring bottleneck link-speed in packet-switched networks," Performance Evaluation, 27\&28, 1996.

[5] M. Coates, R. Nowak. "Network loss inference using unicast end-to-end measurement", Proc. ITC Conf. IP Traffic, Modeling and Management, Sept. 2000.

[6] M.J. Coates and R. Nowak, "Network Delay Distribution Inference from End-to-end Unicast Measurement," to appear in Proc. IEEE ICASSP, May 2001.

[7] A.P. Dempster, N.M. Laird, D.B. Rubin, "Maximum likelihood from incomplete data via the EM algorithm (with discussion)", J. Roy. Statist. Soc. Ser., vol. 39, pp. 1-38, 1977.

[8] A.B. Downey. "Using pathchar to estimate Internet link characteristics," Proc. SIGCOMM'99 Sept. 1999.

[9] N.G. Duffield and F. Lo Presti, "Multicast Inference of Packet Delay Variance at Interior Network Links", in Proc. IEEE Infocom 2000, Tel Aviv, March 2000.

[10] N.G. Duffield, F. Lo Presti, V. Paxson, D. Towsley, "Inferring link loss using striped unicast probes," to appear in Proc. IEEE Infocom 2001, Anchorage, Alaska, April 22-26, 2001.

[11] T. Friedman, R. Cáceres, K. Almeroth, K. Sarac, “RTCP Reporting Extensions”, work in progress, January 2001.

[12] V. Jacobson, Pathchar - A Tool to Infer Characteristics of Internet paths. For more information see ftp://ftp.ee.lbl.gov/pathchar

[13] E.L. Lehmann and G. Casella, "Theory of Point Estimation", Springer Texts in Statistics, Springer, New York, 1998.

[14] R.J.A. Little and D.B. Rubin, "Statistical Analysis with Missing Data”, Wiley, New York, 1987

[15] F. Lo Presti, N.G. Duffield, J.Horowitz and D. Towsley, "Multicast-Based Inference of Network-Internal Delay Distributions", submitted for publication, September 1999.

[16] Mathematica. See www.wolfram.com

[17] G.J. McLachlan and T. Krishnan, "The EM algorithm and extensions”, Wiley, New York, 1997.

[18] ns - Network Simulator. See http://www-mash.cs.berkeley.edu/ns/ns.html

[19] M.J. Schervish, "Theory of Statistics", Springer, New York, 1995.

[20] H. Schulzrinne, S. Casner, R. Frederick, V. Jacobson, "RTP: A Transport Protocol for Real-Time Applications," RFC 1889, January 1996.

[21] Y. Shavitt, X. Sun, A. Wool, B. Yener. "Computing the Unmeasured: An Algebraic Approach to Internet Mapping" to appear in Proc. INFOCOMO1.

[22] C.F. Jeff Wu, "On the convergence properties of the EM algorithm", Annals of Statistics, vol. 11, pp. 95-103, 1982 